\title{
Genf, 1954: Zwischen Ideologie und Realpolitik
}

\section{Die Rolle des sozialistischen Lagers auf der Genfer Indochina-Friedenskonferenz}

Der Kampf gegen den Kolonialismus, der seit 1917 zum ideologischen Gedankengut der russischen Bolschewiki gehört hatte und in den zwanziger Jahren von der Komintern aktiv vorangetrieben worden war, wurde von Stalin weitgehend vernachlässigt. Die Frage, ob die Sowjetunion den antikolonialen Befreiungskrieg unterstützen sollte, tauchte unmittelbar nach dem Zweiten Weltkrieg wieder auf, nachdem die ehemaligen asiatischen Kolonien Großbritanniens ihre Souveränität erlangt und in den Kolonialländern sich mehrere marxistisch geprägte Bewegungen auf der politischen Bühne bemerkbar gemacht hatten. Die Verschärfung des Kalten Krieges zu Beginn der 1950er Jahre ist untrennbar mit dem Dekolonisationsprozess verbunden: Angesichts des Sieges der Kommunisten in China, der Ereignisse in Korea und des Auftretens kommunistischer Parteien in zahlreichen Ländern der Dritten Welt wuchs die Furcht der Amerikaner vor einer kommunistischen Weltherrschaft. Vom Anfang bis zum Ende des Kalten Krieges behinderten die oft von den in Sowjetrussland ausgebildeten Berufsrevolutionären geführten Kriege die Bemühungen um eine Verbesserung der Ost-West-Beziehungen, zunächst die Politik der „Friedlichen Koexistenz““ und dann die Entspannungspolitik. Schon 1953, als sie konzipiert wurde, und somit lange bevor die Chinesen sie zur Streitfrage machten, prallte die Politik der „Friedlichen Koexistenz" mit der Idee der Weltrevolution aufeinander. Der Kreml stand von nun an vor einem unentwirrbaren Dilemma: Einerseits wollte er einen dritten Weltkrieg vermeiden, und dazu musste er irgendeine Form des Kompromisses mit den Vereinigten Staaten finden, andererseits aber war er gewillt, den Kommunismus in der Welt zu verteidigen, zu stärken und zu propagieren.

Dieser Artikel richtet den Fokus auf die Rolle, welche sowohl die UdSSR als auch (hina bei den Verhandlungen über das Genfer Indochina-Abkommen 1954 gespielt haben. Die auf das Drängen Moskaus einberufene Genfer Korea- und Indochinakonferenz veranschaulichen deutlich, wie sich nach Stalins Tod die beiden großen Herausforderungen der Nachkriegszeit - der Kalte Krieg und die Dekolonisation - in der sowjetischen Außenpolitik ineinanderfügten. Zu keinem Zeitpunkt während dieser Zeitspanne stellte für Moskau die Machtübernahme durch ein kommunistisches Regime in Vietnam eine Priorität dar. Gleichwohl taten die Leiter der sowjetischen und der chinesischen Delegation, Molotov und Zhou Enlai ihr Möglichstes, um das Ende des Krieges zu relativ vorteilhaften Bedingungen für den Viet Minh herbeizuführen. Gleichzeitig aber versuchten sie die Eskalation des Konflikts zu vermeiden, der in einen atomaren Krieg hätte ausarten können, da die Vereinigten Staaten sowohl eine militärische Intervention in den Indochinakrieg als auch den Rückgriff auf Präventivschläge gegen die Volksrepublik China nicht nur in Betracht zogen, sondern damit sogar drohten. Noch überraschender ist die Tatsache, dass sie auf den Fortbestand des französischen Einflusses in Indochina großen Wert legten. Beide handelten aus Motivationen heraus, welche den Interessen des Viet Minh in keiner Weise entsprachen: Während Moskau die Unterstützung Frankreichs in Bezug auf europäische Fragen zu gewinnen versuchte, wollte Peking die Errichtung amerikanischer Stützpunkte in beiden Ländern vermeiden. Während die französischen, amerikanischen und sowjetischen Quellen und Memoiren wenigstens teilweise zugänglich sind, sind die vietnamesischen 
Archive bis heute geschlossen. Daher ist es immer noch schwierig, die differenzierten vietnamesischen Zielsetzungen genau zu bestimmen.'

\section{Die „Friedliche Koexistenz” aus der Sicht Moskaus (März 1953-Februar 1954)}

Nach Stalins Tod versuchte die neue sowjetische Führung, die Spannungen in den internationalen Beziehungen zu vermindern, indem sie den Koreakrieg beendete. Sie schlug die Formel der „Friedlichen Koexistenz“ vor und ergriff die Initiative zur Einberufung einer internationalen Konferenz. Gleichwohl blieben die Prioritäten der sowjetischen Außenpolitik unverändert. Europa und insbesondere die „deutsche Frage“ standen weiterhin im Zentrum ihrer Aufmerksamkeit. Im Jahre 1953 scheute Moskau keine Anstrengung, um Frankreich dazu zu überreden, sich dem Projekt der Wiederbewaffnung der Bundesrepublik Deutschland zu widersetzen. Und obwohl sie ihre Waffenlieferungen an Vietnam verstärkte, leistete sie ihm auf diplomatischer Ebene so gut wie keinen Beistand. ${ }^{2}$

Gleich zu Beginn des Jahres 1953 verlagerten sich die internationalen Spannungen von Korea nach Indochina. Gerüchte über geheime sino-sowjetisch-indochinesische Abmachungen alarmierten die amerikanische Regierung. ${ }^{3}$ Am 2. Februar gab der frisch ins Amt eingeführte Präsident der Vereinigten Staaten Dwight David Eisenhower zu verstehen, dass die Vereinigten Staaten sehr wohl auf die Atomwaffe zurückgreifen könnten. Im selben Monat begannen die sowjetischen Behörden die Idee der „Friedlichen Koexistenz“ zwischen dem kapitalistischen und dem kommunistischen System zu fördern, wobei diese Idee von Anbeginn als Alternative gegen einen neuen Weltkrieg gedacht war. So tauchte

\footnotetext{
1 Dieser Artikel ist das Ergebnis der von mir in folgenden Archiven durchgeführten Recherchen: im Archiv des französischen Außenministeriums (Archives diplomatiques françaises - ADF); im Archiv des chinesischen Außenministeriums (Archives du Ministère des Affaires étrangères chinois - AMAEC); in den Unterlagen der Kommunistischen Partei der Sowjetunion, die im Russischen Archiv der Neuesten Geschichte (Rossijskij Gosudarstvennyj Archiv Novejšej Istorii - RGANI) aufbewahrt werden und zum Großteil in den Bibliotheken der Universitäten Yale, Harvard und Stanford verfügbar sind; sowie im Archiv des U.S. Department of State (AUSDS). Ich danke Herrn und Frau Fox, infolge deren Großzügigkeit ich meine Recherchen in Yale als Fox International Fellow fortsetzen konnte, Herrn Christian Ostermann, dem Direktor des CWIHP, der mir liebenswürdigerweise eine Sammlung chinesischer Dokumente, die ins Englische übersetzt wuden, zur Verfügung gestellt hat, die im Bulletin Nr.16 des CWIHP erschienen sind, Herrn Christopher (roscha, der mich über mehrere schwierige Fragen aufgeklärt hat, sowie dem französischen Außenministerium, das mir erlaubt hat, seine Archivbestände zu konsultieren.

$2 \mathrm{Zu}$ Stalins Indochina-Politik siehe Christopher E. Coscha, Courting Diplomatic Disaster? The Difficult Integration of Vietnam into the Internationalist Communist Movement (1945-1950), in: Journal of Vietnamese Studies, Vol.1, No. 1-2, S.59-103; Ilya V. Caaiduk, Confronting Vietnam: Soviet Policy toward the Indochina Conflict, 1954-1963, Washington: Woodrow Wilson Center Press, 2003; Mari Olsen, Soviet - Vietnam Relations and the Role of China, 1949-1954, I ondon/New York 2006.

3 ADF, série (s.) Asie-Océanie, sous-série (ss.) Indochine, dossier (d.) 174 (Communisme. Relations Moscou-Hanoi-Pékin. Août 1944 - mai 1953), S. 269: Titel des Artikels: Accords entre Ho Chi Minh, Moscou et Pékin, in: Franc Tireur, 27.1.1953; die Zeitungen stützten sich auf offizielle französische Quellen, die auf Enthüllungen (nicht nachgewiesen) von Pham Le Bong basieren. Letzterer war ein ehemaliger Viet Minh, der zum I ager Bao Dais übergelaufen ist. ADF, s. Asie-Océanie, ss. Indochine, d. 174, S.271: Artikel von Seymour Freydin betitelt „Stalin's Man of Mystery“, in: Daily Mail, 4.2. 1953. Es handelte sich um Marschall Malinovskij, Oberkommandierender der Roten Armee im Fernen Osten und Georgij Puškin, den stellvertretenden Außenminister der Sowjetunion.
} 
dieser Terminus im offiziellen sowjetischen Sprachgebrauch noch vor Stalins Tod auf und fand fortan ab dem folgenden Monat regelmäßig Anwendung. ${ }^{4}$

Nach Stalins Tod entschied sich die neue sowjetische Führung, Wege zu finden, die zur internationalen Entspannung führen sollten. Als Erstes hatte sie sich zum Ziel gesetzt, dem Koreakrieg ein Ende zu setzen. Am 21. März kündigte das Zentralkomitee der Kommunistischen Partei der Sowjetunion (KPdSU) Zhou Enlai an, welche Absichten es in Bezug auf Korea verfolge. Vier Tage nach seiner Rückkehr in China schlug Zhou Enlai offiziell die Wiederaufnahme der Friedensverhandlungen vor. ${ }^{5}$ Mao akzeptierte das Prinzip der freiwilligen Repatriierung der Kriegsgefangenen - eine Frage, die die Verhandlungen über Korea seit anderthalb Jahren blockiert hatte. Die Initiativen der Sowjets wurden von der chinesischen Führung mit Zustimmung aufgenommen. Sie wollte sich von der koreanischen Last befreien und ihre Energie darauf verwenden, sowohl die Wirtschaft und die Sicherheit ihres Landes zu konsolidieren, als auch einen Sitz in den Vereinten Nationen (UN) sowie eine Einigung über Taiwan zu erreichen. Im Übrigen konnten sie sich der Vaterschaft des Konzepts der „Friedlichen Koexistenz“ rühmen, nachdem Zhou Enlai als Erster im Dezember 1953 dieser entsprechende fünf Prinzipien für die Beziehungen zwischen China und Indien definiert hatte. ${ }^{\circ}$ Die Verhandlungen gingen schnell voran: Am 27. Juli 1953 wurde der Koreakrieg mit dem Waffenstillstand von Panmunjon beendet. Ab diesem Augenblick sollten sich die internationalen Spannungen maßgeblich auf die $\mathrm{Zu}$ kunft Deutschlands und Indochinas auswirken.

Im Gegensatz zu Stalin, der Ho Chi Minh misstraute, hatte die neue sowjetische Führung von ihm ein positives Bild. In seinen Memoiren spricht Chruščev von ihm mit höchst lobenden Worten: Er ging sogar so weit, ihn mit einem jener heiligen Apostel zu vergleichen, die - so sagt man - in längst vergangenen Zeiten lebten:

„Apostel der Revolution [...], seine Augen glänzten vor Aufrichtigkeit und Reinheit. Dabei handelte es sich um die Aufrichtigkeit eines unbestechlichen Kommunisten, der seiner Sache ergeben ist. In Wahrheit war er ein Heiliger. [...] Immer dann, wenn er von dem Kampf sprach, der in Vietnam unter seiner Führung geführt wurde, ja gerade dann, wurde sein auf Stalin und die anwesende Sowjet-Leitung gerichteter Blick etwas eigenartig. Fast - würde ich sagen - lag eine kindliche Naivităt in seinem Blick. ${ }^{\text {77 }}$

Nichtsdestoweniger entwickelte sich die sowjetische Haltung dem Indochinakrieg gegenüber kaum. Im Sommer verstärkte zwar die sowjetische Führung ihre Hilfe für den Viet Minh, proklamierte jedoch weiter ihre Neutralität in dem Konflikt. ${ }^{8}$ Im Frühling 1953 gewann der Krieg an Intensität und breitete sich auf ganz. Indochina aus, was zur Folge hatte, dass die Amerikaner immer mehr an der Fähigkeit Frankreichs zweifelten, den Kommunismus in Asien einzudämmen zu können. So kristallisierte sich die Idee einer nordamerikanischen Intervention in den Indochinakonflikt heraus.

${ }^{4}$ David Holloway, Stalin and the Bomb. The Soviet Union and Atomic Energy, 1939-1956, New Haven/London: Yale University Press, 1994, S. 335.

" King C. Chen, Vietnam and China, 1938-1954, Princeton: Princeton University Press, 1969, S. 283.

"Zhai Qiang, China and the Geneva Conference of 1954, in: The China Quarterly, No. 129 (Mar., 1992), S. 103-122, hier S. 107. Die fünf großen Prinzipien der „Friedlichen Koexistenz:“ sind: die gegenseitige Achtung der Souveränität und der territorialen Integrität, der gegenseitige Nichtangriff, die jeweilige Nichteinmischung in die inneren Angelegenheiten, die Gleichberechtigung und der beiderseitige Nutzen.

${ }^{7}$ N. S. Chruščev, Vremja, ljudi, vlast', Moskau 1999, S.113.

${ }^{8}$ ADF, s. Asie-Océanie, ss. Indochine, d. 174, S.301: Télégramme de Joxe en provenance de Moscou et daté du 9 mai 1953. 
Die neue Sowjetführung bemühte sich um Diversifikation ihrer Außenpolitik, indem sie nicht nur bilaterale Kontakte mit prowestlichen Staaten wie der Türkei, Israel und Österreich zu aktivieren versuchte, sondern auch eine privilegierte Beziehung zu Frankreich anstrebte. ${ }^{9}$ Sehr schnell stellten die französischen Diplomaten fest, dass ihre sowjetischen Amtskollegen ihnen gegenüber eine ungewohnte Freundlichkeit an den Tag legten: Bei jeder Gelegenheit wurde die „französisch-sowjetische Freundschaft“ beschworen. Nach dem Volksaufstand in der DDR im Juni 1953 zeigte sich die sowjetische Führungsspitze dem Projekt einer Europäischen Verteidigungsgemeinschaft (EVG) gegenüber zunehmend feindlicher. Dieses von den Vereinigten Staaten und Großbritannien unterstützte Projekt sah eine Wiederbewaffnung Westdeutschlands unter internationaler Kontrolle vor.

Moskau beschloss die Unterstützung Frankreichs zu gewinnen und gleichzeitig die NATO zu schädigen. Als erstes offenkundiges Zeichen seines Willens zur Annäherung stattete Molotov anlässlich der Feierlichkeiten zum 14. Juli der französischen Botschaft einen unerwarteten Besuch ab, um vier Tage nach Bekanntgabe der Verhaftung Lavrentij Berijas zu signalisieren, dass er die von diesem initiierte „Entspannungspolitik“ fortsetzen würde. ${ }^{10}$ In einer langen Rede am 8. August 1953 vergaß Malenkov, das Indochinaproblem zu erwähnen, was den Franzosen nicht entging. Im Laufe des Sommers sprach der neue sowjetische Botschafter in Frankreich, S. Vinogradov, mehrmals offen über die zwei Leitgedanken, die fortan die sowjetische Außenpolitik maßgeblich prägten. „Wir wollen den Frieden. Die ,Friedliche Koexistenz', so wie sie Lenin definiert hat, gehört zu den Grundsätzen der gegenwärtigen Sowjetpolitik“. Anschließend bestand der Botschafter darauf, dass „die Revolution kein Exportartikel sei“. Diese wirklich revolutionäre Idee wurde natürlich Lenin zugeschrieben. Schließlich schlug der sowjetische Botschafter die Eröffnung direkter Verhandlungen zwischen Frankreich und der Sowjetunion über Deutschland vor.

„Die Existenz eines starken Frankreichs, welches sich seines Indochinaproblems entledigt hat und eben aufgrund dieser Tatsache in der Lage ist, ein eigentlich für Kriegszwecke bereitgestelltes Budget in sozialc Programme $\%$ investicren, erscheint in den Augen der Sowjetunion unverzichtbar: Letztere würde es begrüßen, wenn Frankreich seine traditionelle Mission in vollkommener Eigenständigkeit wieder aufnehmen würde. "II

Es war das erste Mal, dass die Sowjets im Rahmen einer Unterredung mit Frankreich das Indochinaproblem ansprachen. ${ }^{12}$ Aus diesen Signalen schloss Paris, dass die Sowjets „von französischer Seite wenn nicht Unterstützung, so doch wenigstens ein gewisses Entgegenkommen erwarteten“. Es wurde beschlossen, auf diese Annäherungsversuche der Sowjetunion nicht einzugehen. ${ }^{13}$ Ende Oktober bot der sowjetische Botschafter seinem französi-

9 ADF, s. Asie-Océanie, ss. Indochine, d.175) (Communisme. Relations Moscou-Hanoi-Pékin. Juin à décembre 1953), S.51-54: Note de Jean Le Roy à Georges Bidault sur l'URSS et la guerre d'Indochine, datée du 19 août 1953; siehe auch ADF, s. Furope (1945-1955), ss. URSS, d. 154 (Relations entre la France et l'URSS), juillet 1953 - février 1955), S. 54: Note du Consul de France à Malte et à Chypre au Ministre des Affaires étrangères sur la Russie et la France, datée du 15 décembre 1953.

10 ADF, s. Europe (1945-1955), ss. URSS, d. 154, S. 16: Note de Jean Le Roy au Ministre des Affaires étrangères sur l'attitude de l'L'RSS à l'égard de la France faite à Moscou, le 13 août 1953.

11 ADF, s. Secrétariat Général (1945-1966), ss. Dossier, d.31 (Entretien entre Louis Joxe et l'Ambassadeur soviétique en France M. Vinogradov) S.308-316 und 341f., Zitat S. 341 bis: Note de l'Ambassadeur de France en URSS pour le Secrétaire Cénéral faite à Paris le 21 août 1953.

12 ADF, s. Secrétariat (Général (1945-1966), ss. Dossier, d. 31, S.308-316 und 341f.: vgl. Anm. 11.

13 Am 21. August 1953 stellte der französische Außenminister in seinem Brief an den französischen Botschafter in Moskau fest: „Wenn [die Sowjets] darauf aus sind, im Westsystem einen Bruch zu finden 
schen Amtskollegen erneut an, französisch-sowjetische Gespräche zu führen, wobei er ausdrücklich unterstrich, wie gefährlich ihm die Wiederbewaffnung eines „aggressiven und von den Vereinigten Staaten unterstützten Deutschland“ erscheine. Dem Bericht des französischen Botschafters nach zu urteilen, wurde „das Wort vom französisch-sowjetischen Pakt nicht ausgesprochen, aber es schwebte sehr wohl im Raum “. ${ }^{14}$

Parallel zu ihren diplomatischen Bemühungen um Frankreich unternahmen die Sowjets mehrere Schritte sowohl zur Förderung der „Friedlichen Koexistenz“ als auch zur Unterstützung Chinas in seinem Streben, zur Weltmacht aufzusteigen. Am 4. August 1953 schlug die sowjetische Führung den Westmächten vor, eine Fünf-Mächte-Konferenz zur Erörterung der Probleme der Welt einzuberufen, an der auch das bis dahin weder von den Vereinigten Staaten noch von Frankreich anerkannte maoistische China teilnehmen sollte. ${ }^{15}$ Am 24. August 1953 deklarierte Zhou Enlai, dass man sich von der Regelung des Koreakonflikts inspirieren lassen müsse, um alle anderen Konflikte lösen zu können, wobei er in diesem Zusammenhang auf Deutschland und Indochina anspielte. ${ }^{16}$

Die Doktrin der „Friedlichen Koexistenz“ hatte für Moskau sehr gegensätzliche und unerwartete Konsequenzen: Die Beendigung des Koreakrieges trug nicht zu einem Nachlassen der Spannungen zwischen der Sowjetunion und den USA bei. Vielmehr verstärkte sie die amerikanischen Befürchtungen, zusehen zu müssen, wie Indochina und anschließend der ganze asiatische Raum von den Kommunisten einkassiert würden. Als die Chinesen Anfang September ihre 40. Armee in die Nähe der indochinesischen Grenze verlegten, verkündete der amerikanische Außenminister am 2. September, dass eine chinesische Invasion Vietnams „schwerwiegende Konsequenzen haben würde, die sich möglicherweise nicht auf Indochina beschränken würden “. ${ }^{17}$ Im Laufe des Oktobers gab US-Vizepräsident Richard Nixon bekannt, sein Land sei entschieden gegen jegliche Friedensverhandlungen über Vietnam, und am 30. Oktober führte die Eisenhower-Administration ihre Politik des „New Look“ ein. ${ }^{18}$ Im Gegensatz zu dieser Haltung erklärte der französische Premierminister Joseph Laniel vor der Nationalversammlung, dass seine Regierung mit der Idee eines Friedensschlusses einverstanden sei.

Am 26. November ließ der Viet Minh seine Antwort auf Laniels Vorschlag über die Presse verlauten: Ho Chi Minh erklärte seine Bereitschaft zu Waffenstillstandsverhandlungen. Zur gleichen Zeit nahm Moskau von der Idee einer Fünferkonferenz Abstand und schlug

oder gar zu verursachen, wird es für Sie keinen Anlass mehr geben, diese Verhandlungen weiterzuführen.“ADF, s. Europe (1945-1955), ss. URSS, d. 154, S. 22-27, hier S. 27: Minute très secrète du Ministre français des Affaires étrangères à l'Ambassadeur de France à Moscou, Louis Joxe, datée du 21 aô̂t 1953.

14 ADF, s. Secrétariat Général (1945-1966), ss. Dossier, d.31, S.308-316, S.343-348, hier S.344 und 346: Note de l'Ambassadeur de France en URSS pour le Secrétaire Général faite à Paris le 31 octobre 1953.

15 ADF, Secrétariat Général (1945-1966), ss. Conférences Internationales, d.56 (Conférence des Ministres des Affaires étrangères à Berlin, 25 janvier au 24 février 1954, S. 95-104, hier S. 95f.: Note sur la conférence à cinq, datée du 20 janvier 1954.

16 [Ohne Autor] Sụ thật về quan hệ Việt Nam - Trung Quốc trong 30 nam qua [Die Wahrheit über die Beziehungen zwischen Vietnam und China in den letrten Jahren], Hanoi: Nha xuat ban Sự thât, 1979, S. 29.

17 William J. Duiker, US Containment Policy and the Conflict in Indochina, Stanford: Stanford University Press, 1994, S. 144.

18 John Lewis Gaddis, Strategies of Containment. A Critical Appraisal of American National Security Policy during the Cold War, Oxford: Oxford University Press, 2005, esp. ed., S. 147; Duiker, US Containment Policy, S. 132. 
vor, in Berlin eine Konferenz der vier Außenminister über die Frage der Zukunft Deutschlands abzuhalten. Anfang Dezember kamen die westlichen Staatsoberhäupter auf den Bermudas zusammen, um über die sowjetischen Vorschläge zu beraten und ihren politischen Kurs abzustimmen. Diese Gespräche brachten starke Differenzen zum Vorschein. Bidault, der Laniel vertrat, bestand darauf, dass alle Verhandlungen zum Thema Korea Gespräche über Indochina einschließen sollten, wozu die Vereinigten Staaten sich sehr reserviert verhielten, während Churchill den amerikanischen Präsidenten sehr eindrücklich vor den Risiken eines Atomkrieges warnte. ${ }^{19}$ Die Briten fürchteten sich vor den Folgen eines militärischen Eingreifens der USA in Indochina, weil sie annahmen, dass eine solche Intervention zwangsläufig China mit in den Krieg hineinziehen und den Ausbruch eines dritten Weltkrieges nach sich ziehen würde. ${ }^{20}$ Tatsächlich wusste jeder, dass im Februar 1950 Mao Zedong und Stalin einen Freundschafts- und Beistandspakt abgeschlossen hatten. In diesem Abkommen hatten sich die Volksrepublik China und die Sowjetunion gegenseitige Hilfe im Falle eines Angriffs Japans bzw. eines seiner Verbündeten - sprich die Vereinigten Staaten - versprochen. Darüber hinaus sah das Abkommen vor, dass auf alle „zur Verfügung stehenden Mittel zurückgegriffen werden könne“. Dieser Passus, der auf die Tatsache hin deutete, dass die Sowjetunion seit August 1949 im Besitz der Atombombe war, wurde nach langen und schwierigen Diskussionen auf Zhou Enlais Drängen hinzugefügt.

Es herrscht keine Gewissheit darüber, inwieweit die sowjetischen Geheimdienste über die Meinungsverschiedenheiten zwischen den Westmächten hinsichtlich ihrer Haltung zum Kommunismus, zur künftigen Friedenssicherung und zur Wahl der Strategie gegenüber Korea und Indochina unterrichtet waren. Jedenfalls übernahmen die Kommunisten im Januar wieder die Initiative. Am 12. Januar 1954 forderte Zhou Enlai offiziell die Abhaltung einer Fünferkonferenz.. ${ }^{21}$ Am 24. Januar, gleich zu Beginn der Vier-Mächte-Konferenz in Berlin über die deutsche Frage und den österreichischen Staatsvertrag, tat Molotov das Gleiche..$^{22}$ Er legte angesichts der Ablehnung der USA und der zögerlichen Haltung der Franzosen eine ungewöhnliche Entschlossenheit an den Tag. ${ }^{23}$ Ein Beispiel: Anlässlich einer privaten Unterredung mit dem amerikanischen Staatssekretär sprach Molotov fünfzig Minuten lang von der „absoluten Notwendigkeit, sowohl die Rolle Chinas in der Welt als auch den ihm gebührenden Platz. anzuerkennen“. ${ }^{24}$ Er bestand mit solchem Nachdruck auf dieser Forderung, dass sich die vier Außenminister am 18. Februar 1954, also am letzten Tag der Berliner Konferenz, nach erbitterten Gesprächen und dem vollständigen

19 ADF, s. Secrétariat (Yénéral (1945-1966), ss. Dossier, d.31, S. 388-393: Résumé du compte rendu de la séance du 7 décembre aux Bermudes au sujet de la Chine, de la Corée et de l'Indochine, daté du 14 décembre 1953. In einem Anfall von Aufrichtigkeit, der einem die Sprache verschlug, dankte Churchill Bidault „für die Anstrengungen, die Frankreich in Indochina unternahm“, ließ sich aber dann dazu verleiten, die Tatsache zu bedauern, „dass sein I and in Indien nicht gehandelt habe, wie Frankreich es in Indochina tue“.

20) Anthony Eden, The Memoirs. Full Circle, Cambridge 1960, S. 126, 132 und 139.

21 ADF, s. Secrétariat (iénéral (1945-1966), ss. Conférencés Internationales, d. 56, S.95-104, hier S.97: Note sur la conférence à cinq, datéc du 20 janvier 1954.

22 ADF, s. Secrétariat (énéral (1945-1966), ss. Conférences Internationales, d. 56, S. 88-94, hier S.93: Note sur le règlement de la question indochinoise par voie de négociation, datée du 20 janvier 1954. ${ }^{23}$ ADF, s. Secrétariat Général (1945-1966), ss. Conférences Internationales, d.56, S. 200-202: Télégramme de la délégation française signé par Christian de Margerie, daté du 26 janvier 1954.

2.1 ADF, s. Secrétariat Cénéral (1945-1966), ss. Conférences Internationales, d.56, S. 238: Télégramme très secret signé par R. de la Margerie et daté du 30 janvier 1954. Molotovs Äußserungen Dulles gegenüber wurden von Douglas Mac Arthur an die Franzosen weitergeleitet. 
Scheitern der Verhandlungen über die Zukunft Deutschlands und Österreichs darauf einigten, für den 26. April in Genf eine Fünf-Mächte-Konferenz zur friedlichen Regelung des Koreakonflikts und zur Erörterung des „Problems der Wiederherstellung des Friedens in Indochina“ einzuberufen. ${ }^{25}$ Nach der Konferenz hielt Molotov eine Rede, in der er klare Ziele hinsichtlich Koreas setzte: Es müsse „eine nationale Einheit auf demokratischer Basis“ zuwege gebracht werden. Allerdings machte Molotov keine genauen Angaben über seine Absichten in Bezug auf den künftigen Status Indochinas. Er begnügte sich lediglich damit, an die „nationalen Rechte der Völker Indochinas“ zu erinnern und sich für eine Vereinbarung auszusprechen, „die dem Grundsatz der Freiheit und der nationalen Unabhängigkeit der Völker" gerecht werde. ${ }^{26}$

\section{Die kommunistische Strategie am Vorabend der Genfer Konferenz (März-April 1954)}

Während die stets ernsthafte Gefahr einer Eskalation des Krieges bestand, bemühte sich Moskau weiterhin um eine Minderung der internationalen Spannungen und eine in seinem Sinne vorteilhafte Regelung der europäischen Angelegenheiten.

Dementsprechend setzte es seine Verführungspolitik gegenüber Paris fort, dessen Unterstützung es bei den Themen der Zukunft Deutschlands und der Sicherheit in Europa gewinnen wollte. Dennoch vernachlässigte der Kreml keine Minute die kommunistischen Interessen in Asien. Während Paris und Washington über die Bedingungen für einen Kriegseintritt der Vereinigten Staaten hart verhandelten, begaben sich Zhou Enlai und Ho Chi Minh nach Moskau, um Molotov zu treffen, eine gemeinsame Position zu finden und hinsichtlich einer Strategie übereinzukommen. Ziel dieses Treffens war das Zustandekommen einer gemeinsamen Lösung und Strategie. Alle drei zeigten sich sehr interessiert am Erfolg der Genfer Konferenz. Angesichts der Forderungen, die im Rahmen der Plenarsitzung im darauf folgenden Monat formuliert wurden, waren die Beschlüsse, die sie fassten, relativ moderat und lagen nahe bei den am 21.Juli getroffenen Vereinbarungen.

Nach seiner Rückkehr nach Moskau im Anschluss an die Berliner Konferenz erstattete Molotov dem Obersten Sowjet Bericht über die Schritte, die er unternommen hatte. Er präsentierte die Ergebnisse der Konferenz, also die Aufrechterhaltung des Status quo hinsichtlich der deutschen Frage und die Einberufung einer Fünferkonferenz über Korea, als klaren Erfolg der sowjetischen Diplomatie. Besonders freute er sich über die Tatsache, dass China an der Genfer Konferenz teilnehmen und somit „den ihm zustehenden Platz einnehmen wird“ (zakonnoe mesto). Er beschuldigte die Vereinigten Staaten der Vorbereitung eines neuen gegen die Sowjetunion gerichteten Krieges, fügte jedoch hinzu, „dass die von der Sowjetregierung geführte Politik davon ausgehe, dass es keine Streitfragen in den internationalen Beziehungen gebe, die nicht auf friedlichem Wege gelöst werden könnten".27 Molotov verkündete, dass ihm die Berliner Konferenz eine willkommene Gelegenheit zum

\footnotetext{
25 ADF, s. Secrétariat Général (1945-1966), ss. Conférences Internationales, d.56, S. 364: Télégramme de Berlin, 18 février 1954.

${ }^{26}$ ADF, s. Secrétariat Général (1945-1966), ss. Conférences Internationales, d.56, S. 380: Télégramme de Moscou, 6 mars 1954.

${ }^{27}$ RGANI, fond 2, op. 1, delo 89, S. 1-135, hier S. 122: Stenografischer Bericht über das Plenum des ZK der KPdSU, Februar/März 1954. Streng geheim (Bericht Molotovs über die Berliner Konferenz, S. 121-126).
} 
Meinungsaustausch mit dem amerikanischen Außenminister John Foster Dulles über das Thema der Atomwaffen und zur Unterbreitung des Projektes eines internationalen Verzichts auf den Einsatz von Nuklearwaffen geboten habe. Die beiden Männer hätten sich darauf verständigt, in Washington Geheimkonsultationen über dieses Thema zu führen. ${ }^{28}$ Er verunglimpfte die westdeutsche Führung, die er unentwegt mit Nazis verglich. ${ }^{29}$

Das Projekt der Wiederbewaffnung Deutschlands löste bei der sowjetischen Führung eine Unruhe aus, die offenbar nicht vorgetäuscht war. Ein Beispiel: Ende September 1953, anlässlich eines Empfangs in der sowjetischen Botschaft in Paris hatte der sowjetische Botschafter in Frankreich, Vinogradov, Jean Laloy, einem Vertreter des Quai d'Orsay, gegenüber geäußert: „Glauben Sie mir, Sie und ich haben noch einige Zeit zu leben und Sie werden sehen, für Frankreich wird sich Adenauer als noch verhängnisvoller erweisen als Hitler. Auf keinen Fall darf es ein wiedervereinigtes Deutschland mit Adenauer an der Spitze geben." Er hatte erneut für ein Gespräch zu zweit über die deutsche Frage am Rande der Viererkonferenz plädiert. Der Indochinakrieg - für die französische Führung die entscheidende Frage - schien ihn nicht sonderlich zu beschäftigen. Dazu hatte er einfach erklärt: „Sie müssen das bloß selber regeln.“30

In den folgenden Monaten änderten die Sowjets weder ihre Haltung noch ihre Prioritäten: In einer vor dem Obersten Sowjet gehaltenen Rede erwähnte Molotov den indochinesischen Konflikt kaum. Mitte März beschloss die sowjetische Führung, die „Demokratische Republik Vietnam“ zu unterstützen, ohne die Beziehungen zu Frankreich zu beeinträchtigen. In einem vom 17. März 1954 datierenden Memorandum schloss die Sowjetunion explizit aus, die seit Monaten andauernden Bemühungen um eine Vereitelung des Projekts der Europäischen Verteidigungsgemeinschaft zugunsten des Viet Minh aufs Spiel zu setzen. ${ }^{31}$ Am 26. März 1954 empfing der Erste Botschaftsrat der sowjetischen Botschaft in Paris, M. Erofeev, einen Vertreter des Quai d'Orsay und erklärte ihm ohne Umschweife: „Wir versuchen, welchem Krieg auch immer ein Ende zu bereiten. Wir wollen die Entspannung; unser Militärbudget ist schwer zu tragen und wir möchten es deshalb kürzen. Wir stellen keine Verknüpfung zwischen Indochina und anderen Fragen her. Wir wollen zu einem Resultat im Interesse des Friedens kommen. ${ }^{\text {" }}{ }^{32}$ Er meinte wirklich, was er sagte, und Paris schloss mit Recht daraus, dass Moskau einen Modus Vivendi in Indochina erreichen wollte und deshalb nach Mitteln und Wegen suchte, um den ostasiatischen Krieg zu beenden. ${ }^{33}$

Die Chinesen, deren Beziehungen zu Frankreich schwierig waren, zeigten weniger Entgegenkommen. ${ }^{34}$ Mao befürwortete zwar Verhandlungen über Indochina in Genf, war aber der Meinung, dass ihr Erfolg vor allem von einem militärischen Sieg abhing. Er ermunterte die vietnamesischen Kommunisten dazu, ihren Elan auf dem Schlachtfeld zu

${ }^{28}$ Ebenda. Diese Erklärungen sind durch westliche Quellen untermauert.

29 Ebenda.

${ }^{30}$ ADF, s. Asie-Océanie, ss. Indochine, d. 175, S. 278-281, und ADF, s. Europe (1945-1955), ss. URSS,

d. 154, S. 58-61: Note sur l'entretien avec M. Vinogradov, datée du 23 décembre 1953.

31 Olsen, Soviet-Vietnam Relations and the Role of China, S. 32.

32 ADF, s. Europe (1945-1955), ss. URSS, d. 154, S. 84-86: Note datée du 26 mars 1954 sur l'entretien de M. Laloy avec le Conseiller de l'Ambassade d'URSS daté du même jour.

33 ADF, s. Cabinet du Ministre, ss. P. Mendès-France, d. 1, S. 188-196, hier S. 189 bis: Note sur la Conférence de Genève sur l'Indochine du 26 avril au 20 juin 1954.

34 François Joyaux, La Chine et le règlement du premier conflit d'Indochine, Genève 1954, Paris: Publications de la Sorbonne, 1979, S. 175-178. 
steigern, um in die Verhandlungen von einer Position der Stärke aus eintreten zu können. ${ }^{35}$ Den Ratschlägen Maos entsprechend, mobilisierte der Viet Minh in dieser gebirgigen Region enorme militärische Mittel und vor allem immense menschliche Ressourcen. In der Vorbereitungsphase der Konferenz kooperierten die Kommunisten eng miteinander. Auf Veranlassung des Zentralkomitees der KPdSU trafen Präsident Ho Chi Minh, Premierminister Pham Van Dong, der die vietnamesische Delegation in Genf führen sollte, sowie der chinesische und sowjetische Außenminister Zhou Enlai und Molotov am 4. April 1954 in Moskau zusammen, um eine Strategie zu entwickeln. ${ }^{36}$ Zhou Enlai informierte Chruščev darüber, dass zu diesem Zeitpunkt die Kriegslage in Vietnam extrem kritisch war: Die Befreiungsbewegung stehe vor dem Abgrund und Ho Chi Minh habe die Chinesen um direkte Hilfe gebeten. Er beeilte sich jedoch hinzuzufügen, dass China nicht imstande sei, diesem Gesuch nachzukommen, da es Zeit brauche, um nach dem Koreakrieg seine Wirtschaft wieder anzukurbeln. Chruščev hat, nach seiner eigenen Aussage, Zhou Enlai dazu geraten, Ho Chi Minh gegenüber kein Wort darüber zu verlieren. „Zhou stimmte zu, dem Genossen nicht zu sagen, dass China nicht in den Krieg gegen die Franzosen auf vietnamesischem Territorium treten würde. “37 Dem sowjetischen Historiker M. P. Isaev zufolge hat Chou En-lai vorgeschlagen, den Versuch zu unternehmen, in Genf auf direktem Wege mit Frankreich zu verhandeln. ${ }^{38}$ Da die Archive in Vietnam geschlossen sind und infolge des offiziösen Charakters der Memoiren der vietnamesischen Konferenzteilnehmer, ist es weder möglich, die Aussage Chruščevs auf ihren Wahrheitsgehalt hin zu prüfen, noch zu erfahren, in welcher Absicht Ho Chi Minh Zhou Enlai diese vertrauliche Mitteilung anvertraut hätte. Eins ist dennoch sicher: Noch vor dem Beginn der Genfer Indochinakonferenz waren sowohl Sowjetrussland als auch China bestrebt, ein Friedensabkommen zu erzielen.

Dabei favorisierten Sowjetrussland und China die Idee einer Teilung Vietnams nach dem deutschen und koreanischen Modell. Die Teilung entlang der am 16. Breitengrad verlaufenden Demarkationslinie, gegen welche sich der Viet Minh bis Mitte Juli so heftig wehrte, wurde ab Januar 1954 von den Sowjets als eine annehmbare Lösung in Betracht gezogen und von den Chinesen ab März 1954 ebenfalls akzeptiert. Am 6. März erklärte der chinesische Botschafter in einem Gespräch mit Molotov, dass eine solche Teilung für Ho Chi Minh sehr vorteilhaft sei. ${ }^{39}$ In einem vom 2. Mai datierten und von den höchsten chinesischen Instanzen genehmigten Entwurf schrieb Zhou Enlai, dass man, sollte eine Einigung nicht erreicht werden können, zumindest die Möglichkeit haben sollte, weiterhin unter günstigen Bedingungen zu verhandeln, das heißt, indem man einerseits in Indochina den bewaffneten Kampf weiterführte und andererseits sowohl den Konflikt innerhalb der französischen Gesellschaft als auch den zwischen Paris und Washington anfachte. Im Übrigen vertrat er die Ansicht, dass „ein Waffenstillstand vor Ort nicht so gut sei, wie eine am 16. Breitengrad entlang verlaufende Demarkationslinie zwischen dem Norden und dem Süden“, wobei er ausdrücklich darauf hinwies, dass „es zahlreicher Kämpfe bedürfe,

\footnotetext{
35 Yang Kuisong, Changes in Mao Zedong's Attitude toward the Indochina War, 1949-1953, CWIHP, Working Paper No. 34, S. 6.

36 M. P. Isaev/A. S. Černišev, Sovetsko-v’etnamskie otnošenija, Moskau 1975, S.98.

37 Chruščev, Vremja, S. $115 \mathrm{f}$.

${ }_{38}$ Isaev/Černišev, Sovetsko-v'etnamskie otnošenija, S. 98.

39 Gaiduk, Confronting Vietnam, S. 18.
} 
um eine solch günstige Lage zu erreichen “.90 Insgesamt ist festzustellen, dass, selbst wenn sich die drei kommunistischen Alliierten am Vorabend der Genfer Indochinakonferenz darüber einig waren, dass sie gemeinsam nach einem friedlichen Ausgang des Indochinakrieges suchen mussten, sie doch nicht zu denselben Konzessionen bereit waren, um zu diesem einen Ziel zu gelangen. Der Viet Minh - oder zumindest einige seiner Anhänger - hoffte nicht auf eine Teilung Vietnams, sondern auf den vollständigen Rückzug der französischen Truppen aus dem gesamten vietnamesischen Territorium, ferner auf die sofortige Abhaltung von Nationalwahlen - aus denen er zweifellos als Sieger hervorgegangen wäre - und auf die Bewahrung des Einflusses, dessen er sich in den beiden Nachbarländern erfreute. ${ }^{41}$

Am 26. April wurde die Genfer Konferenz eröfnet. Gleich zu Beginn wurden die Verhandlungen über Indochina auf Anfang Mai verschoben. Zu diesem Zeitpunkt war die Lage der französischen Streitkräfte im Kampf um die Dschungelfestung Dien Bien Phu dramatisch geworden. Am 24. April hatte die französische Regierung die USA um sofortiges Eingreifen der amerikanischen Luftwaffe zur Rettung Dien Bien Phus gebeten. Sie bekam eine erneute Absage von Washington, wobei eine spätere Intervention seitens der Amerikaner nicht ausgeschlossen wurde. Am 29. April erklärte Eisenhower den Vereinigten Stabschefs der amerikanischen Streitkräfte, dass die USA „schwerlich der Notwendigkeit ausweichen könnten, zu erwägen, ob man nicht direkt auf den Kopf der Schlange schlagen müsse statt auf deren Schwanz“, wenn es sich bestätigen sollte, dass „Rotchina“ an den Kämpfen in der Region beteiligt war. ${ }^{12}$ Die Gefahr einer Militärintervention der Vereinigten Staaten und somit einer Eskalation des Krieges alarmierte sowohl die Sowjets als auch die Briten. Am Tag vorher, am 5. Mai, hatte Anthony Eden seine Befürchtungen Molotov mitgeteilt: Sollten die Verhandlungen über Indochina scheitern, würden China und die Vereinigten Staaten ihren Kriegseinsatz erhöhen und sich schließlich gegenseitig den Krieg erklären, und dies würde den Beginn eines dritten Weltkrieges bedeuten. Molotov zeigte sich in diesem Punkt vollends mit Eden einverstanden. ${ }^{43}$

Darin liegt möglicherweise der Grund dafür, dass die Beratungen über die Zusammensetzung und die Geschäftsordnung der Kommission in einer kooperativen Atmosphäre stattfanden. Der Viet Minh bestand darauf, dass den Khmer Issarak und dem Pathet Lao - zwei kommunistisch geführte Guerillabewegungen, die unter seiner Kontrolle standen - Vertreter zugestanden werden sollten. Aber Molotov war auf diesem Ohr taub. In seinen ersten Gesprächen mit seinem französischen Amtskollegen Bidault erwähnte Molotov die Khmer Issarak und den Pathet Lao mit keinem Wort. Ebenso wenig bereitete es ihm Schwierigkeiten, von der chinesischen und der Delegation des Viet Minh zu fordern, dass nur Vertreter der Königreiche Laos und Kambodscha sowie des Viet Minh und der südvietnamesischen Regierung unter Bao Dai dazu berechtigt sein sollten, zu den fünf Weltmäch-

40 AMAEC: „Preliminary opinions on the assessment of and the preparation for the Geneva Conference“, prepared by the Ministry of Foreign Affairs Zhou Enlai and approved in principle at a meeting of the C.C.P Central Secretariat, 2 March 1954.

${ }^{41}$ Ebenda, S. 26.

${ }^{42}$ Dwight D. Eisenhower, The White House Years. 1. Mandate for Change, 1953-1956, New York 1963, S. 354

${ }^{43}$ Eden, The Memoirs. Full Circle, S. 132; TASS-Berichte über die Rede Churchills vom 6. Mai, RGANI, f.5, op. 30, d. 29, S. $22-25$. 
ten hinzuzustoßen, was auch letztlich beschlossen wurde ${ }^{44}$ Molotov hielt sich an die Linie, die von der KPdSU am Tag nach der Berliner Vier-Mächte-Konferenz verordnet worden war. ${ }^{45}$ Bidault und Eden akzeptierten ohne Widerspruch, dass der Viet Minh eingeladen werden sollte. Als Gegenleistung allerdings forderten sie am 1. Mai von Molotov und Zhou Enlai, sich beim Viet Minh für die verwundeten Soldaten von Dien Bien Phu einzusetzen. ${ }^{46}$ Am 5. Mai begannen Diskussionen über dieses Thema zwischen französischen und chinesischen Delegierten. ${ }^{47}$ Am 7. Mai ergaben sich zehntausend Soldaten des französischen Expeditionskorps halb verhungert ihren Angreifern vom Viet Minh und beendeten so die 57 Tage währende Belagerung. Am folgenden Tag, dem 8. Mai, wurden die Verhandlungen über Indochina eröffnet. Der aufsehenerregende militärische Sieg erlaubte es dem Viet Minh, die Verhandlungen von einer Position der Stärke aus aufzunehmen, und er konnte auf eine Regelung des Konflikts zu seinen Gunsten hoffen.

\section{Die Konferenz in Gefahr (8. Mai-15. Juni 1954)}

Die in Plenarsitzungen geführten Mai-Verhandlungen erinnern daran, in welchem Maße der Kalte Krieg - in erster Linie - ein Propagandakrieg war, der auf dem Gleichgewicht des Schreckens basierte. Auch wenn Molotov und Zhou Enlai fest entschlossen waren, einen Friedensvertrag abzuschließen, welcher dem Indochinakrieg ein Ende setzen würde, versuchten sie dennoch aus dem internationalen Widerhall, den der Sturz der Festung Dien Bien Phu ausgelöst hatte, Profit zu schlagen. Die Genfer Indochinakonferenz bot ihnen ein außerordentliches Forum, um sich als Verteidiger der unterdrückten Völker darıustellen. Beide fanden indes zu einer wesentlich pragmatischeren Haltung zurück, als die Drohung einer Militärintervention durch die USA sich erneut am Horizont abzeichnete.

Die ersten Plenarversammlungen waren für die Kommunisten die Gelegenheit, ihre Feinde mittels der abgedroschensten sowjetischen Propaganda-Parolen zu schmähen und so die internationalen Spannungen zu verstärken. Sie weigerten sich militärische und politische Fragen getrennt zu behandeln, forderten aber zugleich, dass die politischen Fragen vorrangig behandelt werden sollten, insbesondere der Status von Laos und Kambodscha, und den Platz, der den Aufstandsbewegungen in beiden Ländern eingeräumt werden sollte, festzulegen. Molotov beharrte auf der Notwendigkeit, statt einer Feuereinstellung ein Waffenstillstandsabkommen auszuhandeln, das heißt eine politische Regelung des

it ADF, s. Cábinet du Ministre, ss. P. Mendès-France, d. 1, S. 188-196, hier S. 190 bis: Note sur la Conférence de Genève sur l'Indochine du 26 avril au 20 juin 1954.

45. AMAEC: Télégramme du PCUS au PCC transmis à l'Ambassadeur Yudin, 26 février 1954.

46 AMAEC: Telegram, Zhou Enlai to Mao Zedong and others, regarding a meeting with British Foreign Secretary Eden, 1 May 1954.

${ }^{17} \mathrm{ADF}$, s. Asie-Océanie (1945-1955), ss. Dossiers Généraux, d. 232 (Conférence de Genève, entretiens privés, mai-juillet 1954), S.30-32: Note sur l'entrevue avec le Secrétaire Général de la Délégation chinoise, datée du 6 mai 1954 (siehe auch S.22). - Am 10. Mai kündigte Pham Van Dong an, dass die zum französischen Expeditionskorps gehörenden Soldaten, die verwundet oder krank waren, oder diejenigen Soldaten, die der Armee Bao Dais angehörten und in Dien Bien Phu eingeschlossen waren, befreit werden würden. Siehe AMAEC: Telegram, Zhou Enlai to Mao Zedong and others, regarding the $2^{\text {nd }}$ plenary session, 12 May 1954; ADF, s. Cabinet du Ministre, ss. Georges Bidault (1953-1954), d. 28 (Conférence de Genève du 2 avril au 14 mai 1954), S. 168: Compte rendu sténographique de la deuxième séance plénière tenue au Palais des Nations à Genève le 10 mai 1954, à $15 \mathrm{~h}$. 
Konflikts zu finden. ${ }^{48}$ Molotov machte es sich zur Ehrensache, dass der Indochinakrieg als ein „von Frankreich geführter Kolonialkrieg“ und „vom Viet Minh geführter nationaler Befreiungskrieg“ anerkannt würde. ${ }^{49}$

Die ersten Debatten waren so stürmisch, dass Eden am 13. Mai beschloss, Kontakt mit Molotov und Zhou Enlai aufzunehmen, „um sie darauf hinzuweisen, dass [...], wenn die sowjetische und die chinesische Delegation nichts außer Vorwürfen und Beschimpfungen an die Adresse der Vereinigten Staaten und Frankreichs vorzubringen hätten, diese Provokationen desaströse Konsequenzen haben könnten ". ${ }^{50}$ Eden führte ein Geheimgespräch mit Zhou Enlai, der sich „unflexibel“ zeigte, dann mit Molotov, der „verlegen“ wirkte. ${ }^{51}$ Am folgenden Tag hielt Molotov eine um nichts weniger extrem ideologische Rede. Er verlangte, dass die ,jeweiligen Regierungen der Khmer- und Pathet-Lao-Bevölkerungsgruppen“ angehört würden und dass „der nationale Befreiungskrieg, der in solch großem Umfang in Laos und in Kambodscha geführt würde, nicht unterschätzt werden solle" - zwei Fragen, die er bei den Vorbereitungsgesprächen weitgehend ignoriert hatte. ${ }^{52}$ Das ausgesprochen, ließ die sowjetische Delegation die Franzosen im Verlauf von Geheimgesprächen schleunigst wissen, dass ein sehr allgemeines Grundsatzabkommen über politische Fragen genügen würde..$^{53}$

Am 15. Mai berichteten die Schweizer Morgenzeitungen über die Machenschaften, die sich zwischen Paris und Washington abspielten hinsichtlich eines eventuellen Einschreitens der Vereinigten Staaten in den Krieg. Sollte Eden - zu seiner Überraschung - diese Neuigkeiten aus der Presse erfahren haben, dann liegt der Gedanke nahe, dass es den Kommunisten ebenfalls so ergangen ist. ${ }^{54}$ Zwei Tage später, am 17. Mai, willigte Molotov darin ein, dass die Frage der Zukunft von Laos und Kambodscha getrennt behandelt würde und die militärischen Belange prioritär untersucht würden. Am 19. Mai schrieb Zhou Enlai an Mao Zedong, dass es unmöglich sei, die Position der Westmächte anzunehmen, solange das westliche Lager auf seinem Standpunkt beharre, dass es in Kambodscha und in Laos keine französischen Truppen gebe und dass allein ein Rückzug des Viet Minh es ermögliche, den Konflikt beizulegen. ${ }^{55}$ Dennoch erklärte er sich am 20. Mai bereit, die politischen und militärischen Probleme separat zu behandeln. ${ }^{56}$ Am selben Tag telegrafierte der amerikanische Unterstaatssekretär Walter Bedell Smith an Außenminister Dulles, dass die Kommunisten bereit seien, das Risiko eines militärischen Eingreifens der USA in

4* ADF, s. Asie-Océanie (1945-1955), ss. Dossiers Généraux, d. 232, S. 33-37, hier S. 36: Compte rendu de la réunion franco-anglo-américaine du 6 mai 1954 à $10 \mathrm{~h} 30$.

49 ADF, s. Cabinet du Ministre, ss. (ieorges Bidault (1953-1954), d. 28, S. 258-273, hier S. 259-260 bis: Compte rendu sténographique provisoire, daté du 17 mai 195̃4, de la $4^{\text {ème }}$ séance plénière tenue au Palais des Nations à Genève le 14 mai 1954.

50 ADF, s. Asie-Océanie (1945-1955), ss. Dossiers Généraux, d.232, S.66: Télégramme au départ, envoyé de Genève par G. Bidault le 13 mai 1954.

5 ADF, s. Asic-Océanie (1945-1955), ss. Dossiers Généraux, d.232, S.71-74: Télégramme au départ, très secret, signé de G. Bidault et daté du 14 mai 1954 à 20 h25.

52 ADF, s. Cabinet du Ministre, ss. (Feorges Bidault (1953-1954), d. 28, S. 258-273, hier S. 262 und 266 : vgl. Anm. 49.

53 ADF, s. Cabinet du Ministre, ss. P. Mendès-France, d. 1, S. 188-196, hier S. 194: vgl. Anm. 44.

54 Eden, The Memoirs. Full circle, S. 134.

55 AMAEC: Telegram, Zhou Enlai to Mao Zedong and others, regarding the second restricted session, 19 May 1954.

5j ADF, s. Asie-Océanie (1945-1955), ss. Dossiers Généraux, d.232, S.96f.: Télégramme au départ très secret, daté du 20 mai 1954. 
den Krieg einzugehen ${ }^{57}$, und das amerikanische Militär entwickelte Pläne für eine nukleare Kriegsführung in Indochina.

Die Amerikaner täuschten sich: Die Kommunisten waren nicht bereit, das Risiko einer offenen Konfrontation mit den Vereinigten Staaten einzugehen. Als die Drohung amerikanischer Schläge Gestalt annahm, machten sie mehrere bedeutsame Zugeständnisse. Am 25. Mai nahm Pham Van Dong stillschweigend die Idee einer Teilung Vietnams an. ${ }^{58}$ Am 27. Mai lud ein chinesischer Unterhändler die Franzosen zu direkten Verhandlungen mit der Viet Minh ein und gab sich dabei große Mühe gab, freundlich zu wirken. Er räumte ein, dass „in der Praxis der Fall Vietnams nicht derselbe sei wie der des Pathet Lao und Kambodschas“. ${ }^{59}$ Am 28. Mai entschied sich Washington für eine aktive Verteidigung gegen die Volksrepublik China und jegliche andere von ihr gesteuerte Kraft und fasste den Entschluss, jedweden neuerlichen kommunistischen Vorstoß im südostasiatischen Raum mit einem Gegenschlag auf die militärischen Ressourcen Chinas zu vergelten. ${ }^{60}$ Am 29. Mai, nachdem sie im Rahmen eines verkleinerten Plenums von Zhou Enlai und Molotov kritisiert worden war, erklärte sich die Delegation des Viet Minh damit einverstanden, die Laosund Kambodschafrage gesondert zu behandeln. Auf diese rein formalen Zugeständnisse sollten bald explizitere Erklärungen folgen.

Die Anfang Juni in engstem Kreis geführten Gespräche zeugen von einem Verhaltenswandel, der annehmen lässt, dass die Chefs der kommunistischen Delegationen sich wohl der Ernsthaftigkeit der Gefahr bewusst waren. Anfang Juni reiste Molotov blitzartig nach Moskau, um sich mit seinen Kollegen über den weiteren Verlauf der Genfer Konferenz zu beraten, welche - aus seiner Sicht - an einem kritischen Punkt angelangt war. In diesem Stadium der Verhandlungen hätte sich Moskau mit einem Feuereinstellungsabkommen zufriedengegeben, das es der Sowjetunion erlaubt hätte, ihr Image im Westen zu ändern, ein neues Weltsicherheitsabkommen vorzuschlagen und vor allem das Projekt der Europäischen Verteidigungsgemeinschaft (EVG) zum Scheitern zu bringen ${ }^{61}$ Die Viet-Minh-Delegierten waren trotz eines scheinbar durch nichts zu erschütternden Optimismus sehr beunruhigt ob der Wendung, welche die Konferenz genommen hatte, und glaubten „unisono oder nahezu an eine Gegenoffensive seitens der Vereinigten Staaten in Genf". ${ }^{22}$ Die chinesische Delegation teilte diese Besorgnis. Am 1. Juni führte Zhou Enlai mit Bidault ein Gespräch, zu dem er unmissverständlich sagte, dass seine Hauptsorge einer Verhinderung der Ausweitung des Krieges in Indochina gelte.

„Bei der Konferenz verlangte Pham Van Dong nichts von alledem, was er auf dem Schlachtfeld nicht hätte bekommen können [...]; die reelle Gefahr ist die einer die Sicherheit Südostasiens und Chinas gefährdenden amerikanischen Intervention." ${ }^{\text {i3 }}$

\footnotetext{
${ }_{57}$ AUSDS: Estimate of Duration of Geneva Conference on Indochina. Miscellaneous. Top secret. Issue date: May 20, 1954. Date declassified: June 30, 1981, S. 1 f., hier S. 1.

is Gaiduk, Confronting Vietnam, S.37.

59 ADF, s. Cabinet du Ministre, ss. P. Mendès-France, d. 3 (Conférence de Genève, 1954, négociations Chauvel), S. 3-11, hier S. 4: Note datée du 29 mai 1954 intitulée «nouvelle conversation avec M. Wang Ping Nan, Secrétaire Général de la Délégation chinoise".

${ }^{60}$ Eisenhower, The White House Years. 1. Mandate for Change, S. 361.

${ }^{61}$ ADF, s. Cabinet du Ministre, ss. P. Mendès-France, d. 1, S. 39-41: Note du 4 juin 1954 sur la position de la délégation soviétique à Genève après le voyage de Molotov.

62 ADF, s. Cabinet du Ministre, ss. P. Mendès-France, d. 1, S. 38: Note du 4 juin 1954 sur un renseignement secret du 3 juin concernant les inquiétudes du Vietminh.

63 ADF, s. Asie-Océanie (1945-1955), ss. Dossiers Généraux, d. 232, S. 129-133: Note datée du 2 juin 1954 sur l'entretien de MM. Bidault et Chou En Lai du 1"er juin 1954.
} 
Bidault entgegnete ihm, dass er ebenfalls den Frieden suche, jedoch jegliche Lösung, die mit einer Preisgabe einhergehe, ablehne, und unterstrich in diesem Zusammenhang die prinzipiellen französischen Positionen. ${ }^{64}$

Darauf antwortete ihm Zhou, dass „China überhaupt keinen Krieg wünsche, ob nun einen kalten oder einen heißen“, und lud Bidault ein, eine vertrauensvolle Beziehung zu knüpfen und ihre Anstrengungen zu bündeln. ${ }^{65}$ Am 6. Juni hatte der Generalsekretär der chinesischen Delegation eine ähnliche Unterredung mit dem französischen Unterhändler und Botschafter François Chauvel. ${ }^{66}$

Am 7.Juni, anlässlich einer privaten Unterredung mit Bidault, drückte Zhou Enlai seinen Wunsch aus, dass der Konflikt zwischen dem Viet Minh und Frankreich auf direktem Wege beigelegt werden könne und fügte hinzu: „Wir hoffen auf weiterhin gute Beziehungen zwischen Frankreich und Indochina und wünschen, dass sie sich normalisieren und dass die berechtigten Interessen Frankreichs nach dem Friedensschluss aufrechterhalten bleiben. “67 In Bezug auf den letzten Punkt hielt er sein Wort: Er unternahm alles, um einen Einflussverlust Frankreichs in Indochina zum Vorteil der Vereinigten Staaten zu verhindern.

Trotzdem waren die darauf folgenden Tage von einer deutlich spürbaren und ostentativen Verhärtung der Haltung des kommunistischen Lagers geprägt. Es schien, als ob die Konferenz zu ihren Anfängen zurückgekommen und zum Scheitern verurteilt sei. Am 8. Mai erklärte Molotov im Rahmen einer Plenarsitzung, dass die Einstellung der Kampfhandlungen in Indochina „nicht als eine kurze Atempause betrachtet werden solle, welcher man bedürfe, um den Krieg fortführen und sogar vielleicht ausweiten zu können, sondern als den Auftakt zu einem stabilen Frieden “. ${ }^{8} \$$ Die Laos- und Kambodschafrage rückte wieder ins Zentrum der Debatte. Molotov bestand darauf, dass die Kampfhandlungen, die sich in diesen beiden Ländern abspielten, nicht mehr als Subversionsversuche seitens des Viet Minh betrachtet, sondern vielmehr als „nationale Befreiungskämpfe“ anerkannt werden sollten. ${ }^{69}$

Auf die Stellungnahme des entnervten laotischen Delegierten entgegnete Zhou Enlai, dass es sehr wohl „Widerstandsregierungen“ gebe, zwar nicht in Kambodscha und in Laos, sondern bei den „Khmer und beim Pathet Lao“, und dass es auf keinen Fall in Betracht komme, sie zum Rückzug aufzufordern, da sie autochthon seien, worauf er in scharfen Worten den Vereinigten Staaten die Schuld für die Situation zuschrieb. Obwohl der stellvertretende US-Außenminister zum Gegenangriff gegen die kommunistischen Unterstel-

64 ADF, s. Cabinet du Ministre, ss. P. Mendès-France, d.3, S. 12-19, hier S. 14 und 17: Transcription de l'entretien entre le Président Bidault et M. Chou En I ai du $1^{\mathrm{cr}}$ juin 1954, faite à Genève le 2 juin 1954. Siehe auch die Aufzeichnung des Gesprächs in den chinesischen Akten: AMAEC: Minutes of Zhou Enlai's meeting with Bidault, 1 June 1954.

65 ADF, s. Asie-Océanie (1945-1955), ss. Dossiers Généraux, d. 232, S. 124-128: Projet de télégramme pour Paris sur l'entretien entre le Président Bidault et M. Chou En Lai, daté du 2 juin 1954.

iti ADF, s. Cabinet du Ministre, ss. P. Mendès-France, d. 3, S. 28-35, hier S. 32: Compte rendu daté du 7 juin 1954 de l'entrevue du 6 juin 1954 entre M. (hauvel, Ambassadeur de France en Suisse, et M. Wang Ping Nan, Secrétaire Général de la Délégation chinoise.

${ }_{6 \bar{t}}$ ADF, s. Cabinet du Ministre, ss. P. Mendès-France, d. 1, S. 67-74, S. 73, und ADF, s. Cabinet du Ministre, ss. P. Mendès-France, d. 3, S. 36-43: Note datée du 8 juin 1954 sur l'entretien de MM. Bidault et Chou En Lai le 7 juin 1954.

is ADF, s. Cabinet du Ministre, ss. P. Mendès-France, d. 1, S.91-113, hier S. 101 bis, S. 102 und 104 bis: Compte rendu sténographique provisoire, daté du 11 juin 1954 , de la $5^{\text {ème }}$ séance plénière tenue au Palais des Nations à Genève le 8 juin 1954.

69 ADF, s. Ciabinet du Ministre, ss. P. Mendès-France, d. 1, S.91-113, hier S. 104 bis: vgl. Anm. 68. 
lungen überging, indem er auf die sowjetische Politik vor und während des Zweiten Weltkrieges verwies ${ }^{70}$, beschuldigte Molotov am 10. Juni, die Vereinigten Staaten, die Friedensverhandlungen zu behindern und in Washington Gespräche zu führen, die ein militärisches Eingreifen der USA zum Ziel hatten. Eden jedoch, der für gewöhnlich versöhnlich war, verglich daraufhin „die Aggression“ des Viet Minh gegen Laos und Kambodscha mit den „von Hitler bei der Invasion in die Tschechoslowakei eingesetzten Mitteln“. Erstmals sprach er sich offen für den Rückzug der Truppen des Viet Minh aus diesen beiden Ländern aus. ${ }^{71}$ Pham Van Dong, der bis zu diesem Augenblick eine unerschütterliche Ruhe bewahrt hatte, begann daraufhin einen endlosen Monolog, in dem er die Unabhängigkeit Vietnams und der beiden anderen indochinesischen Staaten, den Rückzug aller ausländischen Truppen aus den drei Ländern und die Abhaltung von Wahlen innerhalb von sechs Monaten forderte. ${ }^{72}$

Die Erklärungen der Kommunisten waren zweifelsohne darauf angelegt, die französische Regierung vor der anstehenden Vertrauensabstimmung der Nationalversammlung in Schwierigkeiten zu bringen. Zweifellos dienten sie ebenso dem Zweck, den Viet Minh in seiner Haltung zu bestärken, der im Begriff war, umfangreiche Zugeständnisse zu machen. Wider Erwarten für die Franzosen wurde an dem Abend jenes strapaziösen 10. Juni einem hochrangigen militärischen Vertreter, Oberst de Brébisson, ein Angebot seitens des Viet Minh unterbreitet, der dabei „eine hektische Eile an den Tag legte“. Das Treffen fand an Ort und Stelle statt. ${ }^{73}$ Tags darauf, am 11. Juni, erklärte Dulles in Los Angeles, dass jedwede mutwillige, offene Aggression Chinas in Südostasien als eine mutwillige Verletzung der nationalen Sicherheit der Vereinigten Staaten betrachtet werden und einen amerikanischen Gegenschlag nach sich ziehen würde. ${ }^{74}$ Am 12.Juni wurde die Regierung Laniel gestürzt. Am selben Tag schlug der Viet Minh heimlich den Franzosen die glatte Teilung Vietnams vor, wobei das gesamte Tonkin ihm zukommen würde.$^{75}$ Am 15. Juni wurden - zu Chou En-lais offensichtlicher Verblüffung - die Verhandlungen über Korea abgebrochen. Aus der Sicht Edens standen zu diesem Zeitpunkt auch die Verhandlungen über Indochina unmittelbar vor dem Scheitern. ${ }^{76}$

\section{Sowjetische und chinesische Bemühungen um eine Regelung des Konflikts (16. Juni-21. Juli 1954)}

Nach dem Scheitern der Korea-Konferenz begann Zhou Enlai die Verhandlungen über Indochina geradezu meisterlich zu führen. Keine Mühe scheuend, machte er zuerst Bidault

70 ADF, s. Cabinet du Ministre, ss. P. Mendès-France, d. 1, S. 114-135, hier S. 127 und 134.

71 AMAEC: Telegram, Zhou Enlai to Mao Zedong and others, regarding the seventh plenary session, 11 June 1954.

72 ADF, s. Cabinet du Ministre, ss. P. Mendès-France, d. 1, S. 136-155, S. 144 bis: Compte rendu sténographique provisoire, daté du 14 juin 1954, de la $7^{\grave{m} m c}$ séance plénière tenue au Palais des Nations à Genève le 10 juin 1954.

73 ADF, s. Cabinet du Ministre, ss. P. Mendès-France, d. 1, S. 188-196, hier S. 195: Note sur la Conférence de Genève sur l'Indochine du 26 avril au 20 juin 1954.

${ }^{74}$ Laurent Césari, La menace d'intervention militaire américaine pendant la Conférence de Genève et la stratégie des Etats-Unis (1954), in: Vingtième Siècle. Revue d'histoire, $\mathrm{n}^{\circ}$ 23, juillet-septembre 1989, S. 3-20, hier S. 13.

75 ADF, s. Ciabinet du Ministre, ss. P. Mendès-France, d. 1, S. 188-196, hier S. 194: vgl. Anm. 73.

76 Eden, The Memoirs. Full Circle, S. 144. 
und dann Mendès-France konkrete Vorschläge, bevor er eine Reise nach Indien und China unternahm. Seine Feindseligkeit gegenüber den Vereinigten Staaten bewog ihn, sich Frankreich gegenüber konziliant $\%$ verhalten und den Erhalt der alten Kolonien in der französischen Einflusssphäre zu befürworten. Molotov, der sich in dieser letzten Verhandlungsphase mehr im Hintergrund hielt, war deswegen nicht minder aktiv und kooperativ. Allein der Viet Minh und die USA versuchten, einer Einigung, die sie als ungünstig einschätzten, entgegenzuwirken.

Nach dem Fehlschlag der Koreaverhandlungen zeigten sich die Kommunisten, die in der Zwischenzeit ihre donnernden Bekenntnisse der letzten Tage vergessen hatten, eifrig daran interessiert, die Diskussion um Indochina neu zu beleben, und machten zu diesem Zweck umfangreiche Konzessionen. Ab dem 15.Juni lockerte Molotov seine Haltung in der Frage der Modalitäten und der Zusammensetzung der Kontrollkommission der neutralen Staaten, zwei Punkte, bei denen er sich bis zu diesem Zeitpunkt unerbittlich gezeigt hatte. ${ }^{77}$ Am 16. Juni teilte Zhou Enlai Eden mit, dass China bereit sei, die Evakuierung der Viet-Minh-Truppen aus Laos und Kambodscha zu akzeptieren und diese beiden Königreiche anzuerkennen, allerdings mit der Bedingung, dass keine nordamerikanischen Stützpunkte in diesen beiden Ländern angesiedelt werden sollten. ${ }^{78}$ Am vorhergehenden Tag hatte der britische stellvertretende Außenminister Harold Anthony Caccia den Chinesen gegenüber wiederholt erklärt, dass es sich bei der britischen Forderung nach einem totalen Rückzug der Viet-Minh-Truppen aus Laos und Kambodscha um eine prinzipielle, nicht verhandelbare Frage handele. Einem französischen Delegierten zufolge wollte „der Viet Minh die Einstellung der Kampfhandlungen. Dieser Wunsch ist sowohl ehrlich als auch kalkuliert." Der Viet Minh, der sich vollkommen im Klaren darüber war, dass die Eroberung des Tonkin-Deltas weit mehr Verluste an Menschenleben verursachen würde als der Kampf um die Festung Dien Bien Phu und dass die Gefahr einer Internationalisierung des Konflikts nicht ausgeschlossen war, schien es eilig zu haben „schnell zu einer Einigung zu kommen ". 79

Am 16. Juni kehrte Bidault, dessen Regierung unterdessen abgesetzt worden war, für einen Tag nach Genf zurück, um mit dem Viet Minh Geheimgespräche zu führen. ${ }^{80}$ Am Tag darauf traf er erneut mit Zhou Enlai zusammen. Dieser teilte ihm mit, dass er den Abbruch der Koreaverhandlungen sehr bedauere und dass er noch vor der Abreise der amerikanischen und britischen Delegationsleiter ein umfassendes Übereinkommen über Indochina erzielen wolle. Nach Aussage Bidaults hat sich Zhou Enlai ,in klaren Worten über Kambodscha und Laos geäußert“ und sowohl „Mäßigung als auch eine hohe Präzision bewiesen“. Er bekräftigte erneut die Notwendigkeit einer gleichzeitigen Einstellung der Feindseligkeiten und einer gemeinsamen politischen Regelung, akzeptierte aber eine unterschiedliche Behandlung der drei Länder. Er räumte offiziell die Anwesenheit von Truppen des Viet Minh in Laos und Kambodscha ein und anerkannte die Notwendigkeit ihrer Evakuierung.

\footnotetext{
77 ADF, s. Asie-Océanie, ss. Dossiers Généraux, d. 209 (Procès-verbaux de la ('onférence de Genève), S.99-101: Propositions de la Délégation de l'URSS présentées à la Conférence de Genève le 14 juin 1954 et traduites du russe.

78 ADF, s. Cabinet du Ministre, ss. P. Mendès-France, d. 1, S. 188-196, hier S. 193 bis: Note sur la Conférence de Genève sur l'Indochine du 26 avril au 20 juin 1954.

79 ADF, s. Asie-Océanie (1945-1955), ss. Dossiers Généraux, d.232, S. 172-177: Note très secrète réservée pour le Président Bidault, datée du 16 juin 1954.

${ }^{80}$ ADF, s. Cabinet du Ministre, ss. P. Mendès-France, d. 1, S. 188-196, hier S. 194: Note sur la Conférence de Genève sur l'Indochine du 26 avril au 20 juin 1954.
} 
Schließlich plädierte er für das Verbleiben der drei indochinesischen Staaten innerhalb der französischen Einflusssphäre.

„Unbekümmert um stilistische Feinheiten, sagte Zhou Enlai, dass Laos und Kambodscha unabhängig, demokratisch und friedliebend sein müssten und in der Union Indochinoise vereint sein sollten. Hinsichtlich Kambodschas, wo die Widerstandsbewegungen begrenæt waren, würde es genügen, wenn die beiden Parteien auf der Stelle die Feindseligkeiten beenden würden. Er wolle berüglich Laos eine Fomel finden, die es erlaube, eine Umgruppierungszone nahe der chinesischen und der vietnamesischen Grenze zu bilden. "\$1

Zhou fügte hinzu, dass China die Regierungen der gegenwärtigen Herrscher wohl anerkennen könne, aber dass man dabei die Existenz der Widerstandsorganisationen in diesen Ländern berücksichtigen müsse. Er machte keinen Hehl daraus, dass er die Schaffung von amerikanischen Militärstützpunkten in Laos und Kambodscha, „die die Sicherheit Chinas bedrohen würden“, sowie die Bildung von Allianzen mit ausländischen Staaten um jeden Preis vermeiden wolle. ${ }^{82}$ In dieser Hinsicht schien ihm Frankreich das beste Bollwerk gegen die Expansion des amerikanischen Einflusses auf der indochinesischen Halbinsel zu sein. Am 18. Juni schrieb er Mao, dass er wünsche, dass „Kambodscha und Laos sowohl Länder südostasiatischen Typs“ werden sollten als auch Mitgliedsländer der Französischen Union. ${ }^{83}$ Am 19. Juni einigten sich beide Delegationen über die Einberufung zweier Militärkommissionen, um die Modalitäten für den Abzug der Viet-Minh-Verbände aus Laos und Kambodscha zu regeln, womit die Präsenz des Viet Minh in diesen beiden Ländern und seine Verstrickung in das Kriegsgeschehen de facto anerkannt wurde. ${ }^{84}$

In der Nacht vom 19. auf den 20. Juni verließen Molotov, Eden und Bedell Smith ${ }^{85}$ Genf. Am 21. Juni führten, im Anschluss an ein chinesisches Abendessen, zu dem die südostasiatischen Delegationen geladen worden waren, der französische Botschafter und Pham Van Dong ein Gespräch unter vier Augen im Hause der chinesischen Delegation, und beide Seiten unterstrichen die Notwendigkeit von geheimen bilateralen Kontakten und einer Annäherung. ${ }^{86}$ Danach führte Chauvel eine Unterredung mit Zhou Enlai, der ihm erklärte, „dass es nicht notwendig sei, lange Reden zu halten, da diese nur eine angespannte Atmosphäre schaffen würden“. Für ihn zählten einzig und allein die unmittelbaren Kontakte: Nur so ließen sich Ergebnisse erzielen. ${ }^{87}$ Am 23. Juni traf Zhou Enlai zum ersten Mal Pierre Mendès-France, den neuen französischen Premierminister, der gleich»eitig das Amt des französischen Außenministers innehatte. Mendès-France hatte sich vorgenommen, innerhalb eines Monats zu einer Einigung zu kommen und sich verpflichtet, zurückzutreten, sollte es ihm nicht gelingen, den Krieg vor dem 20.Juli um Mitternacht zu beenden. Bei dieser Begegnung wiederholte Zhou, was er Bidault am 17. Juni gesagt hatte: China sei gegen jegliche Ausweitung und Internationalisierung des Krieges: dennoch weigere es sich,

${ }^{81}$ ADF, s. Cabinet du Ministre, ss. P. Mendès-France, d.3, S.44-49: Note sur l'entretien du 17 juin entre M. Bidault et M. Chou En Lai. Hervorhebung durch die Verfasserin.

${ }^{82}$ ADF, s. Cabinet du Ministre, ss. P. Mendès-France, d. 1, S. 188-196, hier S. 194: vgl. Anm. 78.

83 AMAEC: Telegram, Zhou Enlai to Mao Zedong and others, regarding Zhou's conversation with Bidault, 18 juin 1954 .

84 ADF, s. Cabinet du Ministre, ss. P. Mendès-France, d. 1, S. 188-196, hier S. 193 bis: vgl. Anm. 78.

ADF, s. Asie-Océanie (1945-1955), ss. Dossiers Généraux, d. 232, S. 178-181, hier S. 180: Note sur l'entrevue entre M. Chauvel et M. Chou En Lai du 22 juin 1954.

${ }^{86}$ ADF, s. Asie-Océanie (1945-1955), ss. Dossiers Généraux, d.232, S.182f: Télégramme au départ très secret envoyé de Genève le 22 juin 1954 et signé de Chauvel sur son entretien du jour avec Pham Van Dong.

87 ADF, s. Asie-Océanie (1945-1955), ss. Dossiers Généraux, d.232, S. 178-181, hier S. 180: vgl. Anm.85. 
zuzusehen, wie Kambodscha und Laos sich in „amerikanische Angriffsbasen“ verwandeln würden. ${ }^{88}$ Er betonte erneut, dass diese Länder weiterhin der Französischen Union angehören sollten. ${ }^{89}$ Daraufhin gab er bekannt, dass Pham Van Dong bereit sei, „die Souveränität , die Unabhängigkeit und die Einheit von Laos und Kambodscha anzuerkennen“, und sprach deutliche Worte über den Rückzug der Viet-Minh-Verbände aus diesen beiden Ländern. Im Gegenzug sollten beide Königreiche die nationalen Guerillaorganisationen anerkennen. Schließlich forderte er Mendès-France auf, sich direkt mit Pham Van Dong zu treffen und sich für eine Kontaktaufnahme zwischen den beiden vietnamesischen Delegationen ohne Einmischung der Vereinigten Staaten zu verwenden. ${ }^{90}$

Die Franzosen waren sehr überrascht darüber, mit welcher Geschmeidigkeit und mit welchem Entgegenkommen Zhou Enlai sowohl über mögliche Konzessionen des Westens als auch über traditionelle Forderungen Chinas hinsichtlich seiner Anerkennung, seiner Haltung zu Taiwan und seiner Mitgliedschaft in den Vereinten Nationen vom Tisch hinwegging. ${ }^{91}$ Nach seiner Unterredung mit Mendès-France verließ Zhou Enlai Genf, um sich nach Indien zu begeben, wo er sechsmal hintereinander mit Nehru zusammentraf. Am 30. Juni ließ der indische Botschafter bei den Vereinten Nationen, Krishna Menon, die französische Regierung wissen, dass sie sich über das Ergebnis dieser Beratungen glücklich schätzen könne, und regte sie dazu an, nach einer Lösung zu suchen, die es den Chinesen und dem Viet Minh erlaube, das Gesicht zu wahren. Seiner Auffassung nach war es nicht notwendig, von ihnen zu fordern, dass sie im französischen Orbit bleiben würden, denn sie würden es auch ohne Aufforderung tun. ${ }^{92}$ Zhou Enlai begab sich anschließend nach Südchina, um sich mit den höchsten Viet-Minh-Führern zu beraten. Leider ist das entsprechende chinesische und sowjetische Archivmaterial noch nicht deklassifiziert. ${ }^{93}$ Am 30. Juni in Guangzhou angekommen, erreichte er Liuzhou am 2. Juli, wo Ho Chi Minh, General Giap, der Botschafter der Demokratischen Republik Vietnam in China, Hoang Van Hoan, sowie der in das Zentralkomitee der ,Partei der vietnamesischen Werktätigen“ entsandte chinesische politische Berater, Luo Guibo, und der chinesische militärische Chefberater der DRV, Wei Guoquing, ihn erwarteten. ${ }^{94}$ Die Lösung, die sich nach einhelliger Übereinstimmung Ende Juni abzuzeichnen schien, sah in territorialer Hinsicht die Teilung Viet-

\footnotetext{
${ }^{88}$ ADF, s. Cabinet du Ministre, ss. P. Mendès-France, d.3, S. 50-55, hier S. 51: Télégramme urgent et très secret, signé de Jacques Roux et envoyé de Genève le 24 juin à $4 \mathrm{~h}$, au sujet de l'entretien entre Chou En Lai et Pierre Mendès-France.

89 AMAEC: Minutes, Zhou Enlai's meeting with Mendès-France, 23 June 1954.

${ }^{90} \mathrm{ADF}$, s. Cabinet du Ministre, ss. P. Mendès-France, d. 3, S. 50-55, hier $\mathrm{S.} .51$ und 53 bis: Télégramme urgent et très secret, envoyé de Genève le 24 juin à $4 \mathrm{~h}$, au sujet de l'entretien entre Chou En Lai et Pierre Mendès-France.

${ }^{91}$ ADF, s. Cabinet du Ministre, ss. P. Mendès-France, d.3, S. 57: Télégramme secret du 25 juin 1954 destiné à Londres et à Washington.

${ }^{92}$ ADF, s. Cabinet du Ministre, ss. P. Mendès-France, d. 2 (Conférence de Genève du 22 mai à la fin juin 1954 - suite), S. 16f.: Télégramme adressé de New York et daté du 1'r juillet 1954.

93 AMAEC: Telegram, CC.P Central Committee to chief military advisor to the Vietnamese Worker's Party Wei Guoqing, Qiao Xiaoguang and conveyed to the VWP Central Committee, regarding the meeting between the Premier and Comrade Ding [Ho Chi Minh's pseudonym], 20 June 1954. AMAEC: Telegram, Zhou Enlai to Mao Zedong, Liu Shaoqi and the CCP Central Committee, ,a brief report on the meetings at Liushou", $1.00 \mathrm{pm}, 3$ July 1954.

${ }^{94}$ AMAEC: Telegram, Zhou Enlai to Mao Zedong, Liu Shaoqi and the CCP Central Committee, „a brief report on the meetings at Liushou", $1.00 \mathrm{pm}, 3 \mathrm{July}$ 1954. Zum Thema der chinesischen Berater in der DRV vgl. Christopher Goscha, Historical Dictionary of the Indochina War (1945-1954), Lanham (in Vorbereitung).
} 
nams, den vollständigen Abzug der Viet-Minh-Truppen aus Kambodscha und deren Teilabzug aus Laos vor, in militärischer Hinsicht das Verbot der Errichtung amerikanischer Militärbasen in Laos und Kambodscha, sowie das eventuelle Verbleiben französischer Truppen in diesen beiden Ländern, und schließlich in politischer Hinsicht die Koexistenz von zwei provisorischen Regierungen in Vietnam und die schnellstmögliche Abhaltung von Wahlen. Die Franzosen strebten nach einem Übereinkommen, welches dem südvietnamesischen Staat „ein möglichst nach allen Seiten wasserdichtes Territorium“ zugestehen und gleichzeitig den zivilen Frieden sowie die Sicherheit durch die Präsenz des französischen Expeditionskorps garantieren sollte. Als Entschädigung für die Evakuierung des Tonkin wollten sie eine Einigung über die Waffenruhe erzielen, ferner strebten sie die Festsetzung der Vietnam teilenden Demarkationslinie vor den Toren Annams, das heißt in der Nähe des 18. Breitengrades, an sowie klare Positionen in Bezug auf den Hafen von Hai Phong, hinsichtlich Fristen innerhalb derer und die Bedingungen unter denen die Wahlen stattfinden sollten, und des Schicksals der Bistümer Bui Chu und Phat Diem, ferner ging es ihnen um Garantien für die Wahrung ökonomischer Interessen Frankreichs im Tonkin. ${ }^{95}$ Die Franzosen zeigten sich über die "gravierenden Auswirkungen“ besorgt, welche die in Genf getroffenen Beschlüsse für Indochina haben könnten, insbesondere die Ankündigung der Teilung des Landes, welche schwere Unruhen - politische wie militärische - im franco-vietnamesischen Lager auslösen könne. Dazu zählten fremdenfeindliche Massenunruhen, Feindseligkeiten der vietnamesischen Armee gegen das französische Expeditionskorps oder gar Verrat seitens der in das Expeditionskorps integrierten Vietnamesen..$^{96}$

Den Memoiren eines erstrangigen chinesischen Delegierten zufolge hat Zhou Enlai Pham Van Dong gebeten, wegen des 16. oder 17. Breitengrades nicht herumzufeilschen. Seiner Meinung nach stand diese Konzession in keinem Verhältnis zu derjenigen der Franzosen, die sich bereit erklärt hatten, sich aus Tonkin zurückzuziehen, und gab dem französischen Premierminister die Möglichkeit, sein Gesicht zu wahren. Zhou habe hinzugefügt: „Nach dem Rückzug der Franzosen wird ganz Vietnam Ihnen gehören. ${ }^{\text {"97 }}$ Folglich hatten die Chinesen zu keiner Zeit - weder vor noch während der Konferenz - die Absicht gehabt, Druck hinsichtlich des 13. Breitengrades auszuüben. Was Mendès-France anbetraf, hatte er Chauvel gebeten, auf Pham Van Dong zuzugehen, um direkte Verhandlungen über territoriale Fragen einzuleiten. ${ }^{98}$ Am 25.Juni 1954 traf Chauvel Pham Van Dong, „der sich direkten Verhandlungen mit der Regierung von Bao Dai“ nicht widersetzte. ${ }^{99} \mathrm{Jedoch}$ beklagte sich Chauvel am 26.Juni bei Vasilij Kuznecov darüber, dass das morgendliche Treffen der französischen und der Viet-Minh-Militärexperten zu keinem Ergebnis geführt habe. Während Pham Van Dong bereits zweimal hintereinander - nämlich am 22. und am 25.Juni - die Trennung der politischen und militärischen Fragen akzeptiert habe, verlang-

95 ADF, s. Asie-Océanie (1945-1955), ss. Dossiers Généraux, d. 232, S. 184: Fiche à l'attention de Monsieur l'Ambassadeur Chauvel émanant de la Commission militaire française à Genève et datée du 23 juin 1954.

96 ADF, s. Asie-Océanie (1945-1955), ss. Dossiers Généraux, d. 232, S. 185-187: Note n 15, très secrète, de la Commission militaire française à Genève à l'attention de Monsieur l'Ambassadeur Chauvel, datée du 23 juin 1954.

97 Zhai Qiang, China and the Geneva Conference of 1954, S. 111.

98 ADF, s. Cabinet du Ministre, ss. P. Mendès-France, d.2, S. 77f.: Télégramme daté du 26 juin 1954 , signé par Pierre Mendès-France et envoyé à Londres et Washington.

99 ADF, s. Asie-Océanie (1945-1955), ss. Dossiers Généraux, d. 232, S. 192-194, hier S. 193: Télégramme secret daté du 25 juin 1954 et signé de Chauvel au sujet de son entretien du jour avec Pham Van Dong. 
ten die militärischen Delegierten des Viet Minh „eine von einem französischen Politiker verfasste Gesamterklärung "100, bevor man fortschreiten könne. Am frühen Abend des 26. Juni kamen die chinesische, sowjetische und die Viet-Minh-Delegation zusammen, um ihre Positionen aufeinander abzustimmen. Die Vietnamesen legten ihren Plan vor: In Bezug auf Laos sollten die französischen Truppen aus dem Norden und die kommunistischen Verbände aus dem Süden bzw. aus der Landesmitte abziehen. Im Falle Vietnams sollte die Demarkationslinie im günstigsten Fall zwischen dem 13. und 14. Breitengrad und schlimmstenfalls nahe dem 16. Breitengrad festgesetzt werden. Novikov, der sowjetische Delegierte, verbarg seine Unzufriedenheit nicht angesichts der Ungenauigkeit der VietMinh-Vorschläge zur Abgrenzung der Umgruppierungszonen. Er bat Pham Van Dong, „die Lage weiter zu untersuchen und dann konkrete Vorschläge zu machen“.101 Die von Chauvel formulierten Vorwürfe blieben offenbar nicht ohne Wirkung.

Die Sowjets waren umso geneigter, die französischen Unterhändler zufriedenzustellen, als Letztere alles unternahmen, um ihnen entgegenzukommen. Besonders konziliant zeigten sie sich bei der Aushandlung der Bedingungen, unter denen die internationale Kontrolle, derer sich die Sowjets schon von Anfang an angenommen hatten, durchgeführt werden musste. In einem am 1.Juli schriftlich festgehaltenen Bericht unterstrich der französische Delegierte Offroy, dass die Formulierung der sowjetischen Forderungen „durch die unverkennbare Sorge um Ausgleich geleitet worden sei und nachdem substantielle Zugeständnisse gemacht worden seien“, während die Nordvietnamesen sich gerade in dieser Phase als kompromisslos erwiesen hatten. ${ }^{102}$ Sie versuchten, die Verhandlungen hinauszuschieben, bis sie über die Ergebnisse des Treffens zwischen Zhou Enlai und Ho Chi Minh verfügen konnten. Sie stellten weiterhin den Anspruch auf eine Beteiligung der Vertreter der Aufstandsbewegungen aus Laos und Kambodscha, die Festlegung der Demarkationslinie entlang dem 13. Breitengrad, die Durchführung allgemeiner freier Wahlen binnen eines halben Jahres zwecks der Wiedervereinigung des Landes sowie die Schaffung zweier Umgruppierungszonen für die ,roten Laos-Partisanen“. Am 9. Juli informierte Chauvel Molotov darüber, dass die Laos-Verhandlungen aufgrund der überzogenen Ansprüche des Viet Minh und aufgrund der Vorbehalte der laotischen Regierung auf der Strecke geblieben seien. Ebenso beklagte er sich über die mangelnde Kooperationsbereitschaft der militärischen Berater Vietnams, die gerade eine Laos-Karte angefertigt hatten, in der das laotische Territorium in zwei Hälften geteilt worden war, um dem Pathet Lao eine Hälfte zuzuteilen. ${ }^{103}$

Vietnamesischen Quellen zufolge soll Zhou Enlai folgendes Telegramm an zwei chinesische Berater gesendet haben, die zum Zentralkomitee der Partei der vietnamesischen Werktätigen - so nannte sich die kommunistische Partei seit 1951 - abgeordnet worden waren, erstrangige politische und militärische Funktionen innehatten und sich eines groBen Einflusses bei den vietnamesischen Kommunisten erfreuten:

\footnotetext{
${ }^{100}$ ADF, s. Asie-Océanie (1945-1955), ss. Dossiers Généraux, d. 232., S. 195-199, hier S. 197: Entretien de M. Chauvel avec M. Kouznetsov, tenu à Genève le 26 juin 1954.

101 AMAEC: Telegram, Li Kenong to Mao Zedong, Liu Shaoqi, and the CC.P Central Committee, and conveyed to Zhou Enlai, Zhang Wentian and Wang Jiaxiang, concerning the content of a meeting between Soviet, Chinese and Vietnamese delegations, 26 June 1954.

102 ADF, s. Asie-Océanie (1945-1955), ss. Dossiers Généraux, d.232, S.211-216, hier S.216: Note $\mathrm{n}^{\circ} 999$ réservée, faite à Genève le $1^{\text {cr }}$ juillet 1954.

${ }^{103}$ ADF, s. Asie-Océanie (1945-1955), ss. Dossiers Généraux, d. 232, S. 232-236.
} 
„Es sind gerechte und rationelle Voraussetzungen dafür geschaffen, dass die französische Regierung [dem Abschluss der Vereinbarung] innerhalb von zehn Tagen zustimmt. Die vorgebrachten Bedingungen müssen klar und einfach sein, um den Verhandlungsprozess zu begünstigen. Man darf sie nicht in komplizierter und verwirrter Form übergeben, möchte man Zeitverlust bei den Verhandlungen, eine Überfrachtung und Verlängerung vermeiden sowie die Sabotierung durch die Vereinigten Staaten [verhindern]." ${ }^{104}$

Diese Botschaft kann als eine verdeckte Aufforderung interpretiert werden, Druck auf die Viet-Minh-Führung auszuüben und von ihr zu fordern, dass sie ihre Delegation in Genf entsprechend instruiere. Am Abend des 10. Juli trafen Molotov und Mendès-France zu einem Abendessen zusammen. Bei dieser Gelegenheit erklärte der französische Premierminister, warum ihm der 18. Breitengrad als die bequemere Lösung erscheine, welche den Interessen beider Parteien am meisten gerecht würde. Vor den Toren Annams verlaufend, „stelle sie eine historische, ethnische und politische Grenze dar“. Sie sei „kurz und daher passend, um die Überwachung und die Truppenstationierung zu vereinfachen“. Molotov antwortete darauf, dass der Viet Minh vorgeschlagen habe, die Demarkationslinie „in der Nähe des 14. bzw. 13. Breitengrades zu ziehen“, fügte jedoch hinzu, dass der Viet Minh mittlerweile Vorschläge gemacht habe, die dazu tendierten, die Demarkationslinie weiter in den Norden zu verschieben. ${ }^{105}$ Laut Molotov blieb der Viet Minh bis zum 12. Juli bei dieser Position. ${ }^{106}$

Am 13.Juli stimmte Pham Van Dong im Anschluss an eine Unterredung mit Zhou Enlai, der am Tag vorher in Genf angekommen war, der Festsetzung der Demarkationslinie entlang dem 16. Breitengrad zu. Allerdings beharrte er auf der Teilung Laos und der Schaffung einer temporären militärischen Umgruppierungszone für das Pathet Lao. ${ }^{107}$ Am selben Tag deutete Zhou Enlai im Laufe einer privaten Unterredung mit Mendès-France an, dass der Viet Minh noch größere Zugeständnisse machen könnte. Mendès-France ergriff die Gelegenheit und schlug ihm vor, dass auf eventuelle Konzessionen zur Demarkationslinie das andere Lager mit anderweitigen Konzessionen reagieren solle. Das „Opfer von Hanoi“ sei wichtiger als das, zu dem der Viet Minh sich bereit erklärt habe, als er die von ihm kontrollierte Zone in der Nähe des 15. Breitengrades aufgegeben habe, und sei es nur, weil es die Evakuierung von 300000 Personen, gegenüber 30000 für den Viet Minh erfordert habe. Zhou zog daraus folgendes Fazit: „Wenn Frankreich ein wenig nachgebe, wird die DRV viel nachgeben; und ich wiederhole: Ich glaube zu wissen, dass Herr Pham Van Dong Sie vor Ihrer Abreise nach Paris gerne sehen würde. "108 Etwas später im Laufe des Tages ließ er Eden wissen, dass die Frage um Kambodscha bald gelöst sein werde und dass die Laosfrage es auch bald sein könne, dies allerdings nur unter der Bedingung, dass es weder ausländische Militärstützpunkte noch Militärbündnisse mit den Vereinigten Staaten in diesen beiden Länder gebe. ${ }^{109}$

\footnotetext{
104 Sự thật về quan hệ Việt Nam - Trung Quồc, S. 32.

105 ADF, s. Asie-Océanie (1945-1955), ss. Dossiers Généraux, d. 232, S. 237-243, hier S. 238f.: Entretien de Mendès-France avec Molotov, Genève, le 12 juillet 1954.

${ }^{106}$ ADF, s. Asie-Océanie (1945-1955), ss. Dossiers Généraux, d.232, S. 244: Télégramme daté du 13 juillet 1954 et signé par Mendès-France concernant deux entretiens du 12 juillet avec $M$. Eden.

${ }^{107}$ Lưu Văn Lợi/Nguyền Hồng Thạch, Pháp tái chiềm Đông Dương và chiến tranh lạnh [Die französische Wiederbesetzung Indochinas und der Kalte Krieg], Hanoi 2002, S. 247.

${ }^{108} \mathrm{ADF}$, s. Cabinet du Ministre, ss. P. Mendès-France, d.5, S. 47-53, hier S. 49: Entrevue de MendèsFrance avec Chou En Lai, le 13 juillet 1954. ADF, s. Cabinet du Ministre, ss. P. Mendès-France, d. 3, S.68f.: Télégramme réservé envoyé de Genève par Chauvel, le 13 juillet 1954.

${ }^{109}$ AMAEC: Minutes, Zhou Enlai's meeting with A. Eden, 13 July 1954.
} 
Noch am selben Tag bekundete der amerikanische Außenminister seine Bereitschaft, nach Paris zu kommen, um Mendès-France und Eden zu treffen. ${ }^{110}$ Weder Dulles noch sein Stellvertreter Bedell Smith waren nach Genf zurückgekehrt, weil sie fürchteten, sie müssten sich öffentlich von den Ergebnissen der Konferenz distanzieren. Das Treffen fand am 14. Juli in Paris statt, was laut Eden Molotov in hohem Maße verärgerte. Mendès-France und Dulles unterschrieben ein Sieben-Punkte-Memorandum, das mit jenem vergleichbar war, welches von den Briten und den Amerikanern Ende Juni in Washington unterzeichnet worden war. Dieses Dokument, das dem Zweck diente, Frankreich an das westliche Lager zu binden und seinen Handlungsspielraum im Genfer Verhandlungsprozess einzuschränken, sah insbesondere vor, dass die abschließende Übereinkunft sowohl die territoriale Integrität und die Unabhängigkeit von Laos und Kambodscha als auch den Rückzug der Viet-Minh-Verbände aus diesen beiden Ländern garantieren müsse. ${ }^{111}$ Diese neuerlichen Gespräche im westlichen Lager beunruhigten die Kommunisten zweifellos.

Am selben Tag, in Genf, hatte der französische Diplomat und Chinaexperte Jacques Guillermaz während seines Treffens mit Wang Ping Nan das Gefühl, dass die chinesische Seite es für „äußerst wünschenswert halte, die Konferenz zu einem Ergebnis zu führen, ganz gleich, ob der amerikanische Außenminister anwesend sei oder nicht", wobei er anmerkte, dass die Losung nicht „Unnachgiebigkeit“ sondern „Sympathiebezeugung“ lautete. ${ }^{112}$

Am 16. Juli überbrachte Mendès-France Molotov und Eden die Nachricht, dass der Viet Minh seine Einwilligung zur Festlegung der Demarkationslinie entlang dem 16. Breitengrad gegeben habe. Molotov forderte ihn auf, als Gegenleistung dem Viet Minh ein ähnliches Zugeständnis zu konzedieren, indem er den 16. anstelle des 18. Breitengrades akzeptiere. Mendès-France lehnte strikt $a b$, indem er daran erinnerte, wie vorteilhaft der Verzicht auf das Delta des Roten Flusses für den Viet Minh sei, und mit der Frage endete, „weshalb denn der Viet Minh auf einer Route bestehe, die zwar keine Region Vietnams versorge“, aber nach Laos führe. ${ }^{113}$ Molotov warf einige weitere Fragen auf, insbesondere diejenige der Frist, innerhalb derer die Truppenumgruppierung erfolgen sollte, und plädierte für einen Zeitraum von sechs Monaten, die Nichteinführung von Kriegsmaterial und Truppen sowie das Verbot, militärische Stützpunkte zu errichten und militärische Allianzen einzugehen. Besonderen Wert legte er auf das Datum der Wahlen, die er im Juni 1955 oder spätestens im Laufe des Jahres 1955 abgehalten haben wollte. Am selben Tag verkündete der Viet Minh sein Einverständnis mit Frankreichs Position zur Frage der internationalen Kontrolle über die Durchführung des Waffenstillstandsabkommens: Diese sah vor, dass in jedem Land eine Kommission gebildet werden solle, deren Aktivitäten von einem Zentralorgan koordiniert werden sollten. ${ }^{114}$ Am 17. Juli beschwerte sich Mendès-

${ }^{110}$ ADF, s. Cáabinet du Ministre, ss. P. Mendès-France, d. 3, S. 115-117: Télégramme signé de Bonnet et daté du 13 juillet 1954 .

111 AUSDS: Confidential memorandum, France-United-States position paper on Indochinese accords. Issue date: July 14, 1954. Date declassified: November 15, 1982.

112 ADF, s. Cabinet du Ministre, ss. P. Mendès-France, d. 3, S. 70-77, hier S. 71: Note pour l'Ambassadeur Chauvel faite à Genève le 14 juillet 1954 au sujet de l'entretien du même jour entre Guillermaz et Wang Ping Nan.

${ }^{113}$ ADF, s. Asie-Océanie (1945-1955), ss. Dossiers ('énéraux, d. 232, S. 254-263: Entretien des ministres français, anglais et soviétique du 16 juillet 1954; compte rendu fait à Genève le 17 juillet 1954.

114 ADF, s. Asie-Océanie (1945-1955), ss. Dossiers Généraux, d. 232, S. 264-266: Entretien des ministres américain, britannique et français daté du 17 juillet 1954. 
France bei Zhou Enlai über die mangelnde Kooperationsbereitschaft des Viet Minh in der Laosfrage und legte ihm anschließend nahe: „Geben Sie doch Herrn Pham Van Dong einen Rat, wie Sie es bei vielen Anlässen bereits getan haben, und verlangen Sie von ihm, er möge realistischere Überlegungen anstellen." ${ }^{115}$ Mendès-France brachte auch gegenüber Molotov und Eden seine Verärgerung zum Ausdruck und beklagte insbesondere, dass die französische Delegation seit Tagen die Offiziere des Viet Minh nicht mehr zu Gesicht bekommen habe. Am 16. Juli hatten sich die Kommunisten darauf verständigt, dass Pham Van Dong auf der Festlegung sowohl der Demarkationslinie entlang des 16. Breitengrades als auch eines Wahltermins bestehen würde. ${ }^{116}$ Die einzige Antwort Molotovs darauf war, „er bekenne, nichts über die Gründe zu wissen, welche ein Zusammentreffen der französischen und der Viet-Minh-Experten verhindert hätten, da Herr Pham Van Dong ihn noch nie auf diesbezügliche Schwierigkeiten angesprochen habe“. ${ }^{117}$ Ebenfalls an diesem 17.Juli versicherte Zhou Eden, dass die chinesische Haltung in der Laosfrage seit seinem Vorschlag vom 16. Juni sich nicht geändert habe, fügte jedoch hinzu, dass er sein Versprechen halten würde. Hinterher bat Zhou Eden, ihm Aufschluss über das von den Amerikanern geplante südostasiatische Verteidigungsprojekt (die dann im September 1954 gegründete SEATO - Southeast Asia Treaty Organisation) zu geben. Er wollte wissen, ob sie die Errichtung amerikanischer Basen in Indochina oder den Abschluss eines Militärbündnisses mit diesen Ländern beabsichtigten. Eden antwortete: „Die Amerikaner haben mir gesagt, dass sie nicht die Absicht hätten, Militärbasen in Laos und Kambodscha einzurichten. Natürlich, falls der Viet Minh Laos oder Kambodscha einnehmen wolle, bevor oder nachdem ein Abkommen erzielt werden kann, dann werden wir und die USA unsere Besorgnis zum Ausdruck bringen." ${ }^{118}$ Noch am selben Tag übte Zhou im Rahmen eines Gesprächs mit Mendès-France Druck dahingehend aus, dass Laos und Kambodscha keine Allianz mit den Vereinigten Staaten schließen sollten.

Am Morgen des 18.Juli beauftragte Eden seinen Stellvertreter, Caccia, den Chinesen zu sagen, dass auf indochinesischem Boden kein militärischer Stützpunkt das Licht der Welt erblicken werde, und dass diese Länder sich nicht der SEATO anschließen würden, wenn ein für alle Konferenzteilnehmer annehmbares Waffenstillstandsabkommen zustande käme. ${ }^{119}$ Noch am selben Tag schrieb Eden nach London, seiner Meinung nach betrage die Chance, dass die Konferenz zu einem erfolgreichen Abschluss komme, nicht mehr als 50 Prozent. ${ }^{120}$ Aber er hatte nicht mit der Entschlossenheit der Kommunisten gerechnet. Molotov versprach Mendès-France in Gegenwart Edens, seinen Plan zu unterstützen. Später am Abend informierte Zhou Enlai Eden, dass er mit der Zusammensetzung der Kontrollkommission aus Indien, Polen und Kanada einverstanden sei. Die Verhandlungen waren zwar beträchtlich vorangekommen, stießen allerdings immer noch auf drei große Hindernisse: die Demarkationslinie, den Wahltermin und die Laosfrage.

Am 19. Juli gaben die chinesischen Delegierten Wang Ping Nan und Zhang Wentian einem französischen Delegierten bzw. Caccia bekannt, dass die Viet-Minh-Delegation zwei

\footnotetext{
115 AMAEC: Minutes, Zhou Enlai’s meeting with Mendès-France, 17 July 1954.

116 Gaiduk, Confronting Vietnam, S. 46.

11 ADF, s. Asie-Océanie (1945-1955), ss. Dossiers Généraux, d. 232, S. 267-271, hier S. 269: Entretien des ministres français, anglais et soviétique le 17 juillet 1954.

118 AMAEC: Minutes, Conversation between Zhou Enlai and Eden, 17 July 1954.

119 AMAEC: Telegram, Zhou Enlai to Mao Zedong and others, regarding the situation at the twentythird restricted session, 19 July 1954.

${ }^{120}$ Gaiduk, Confronting Vietnam, S. 47.
} 
zusätzliche Zugeständnisse gemacht habe: Sie seien bereit, „die Demarkationslinie 10 Kilometer nördlich von der Fernstraße Nummer 9 festzulegen, und die Wahlen innerhalb von zwei Jahren zu organisieren“.'121 Der Einschätzung zweier vietnamesischer Zeithistoriker zufolge wurden diese beiden Konzessionen auf Initiative der Volksrepublik China gemacht - möglicherweise ohne die Zustimmung des Leiters der Viet-Minh-Delegation. ${ }^{122}$ Am selben Tag sprach Zhou mit Eden und Mendès-France über Laos. Er forderte erneut, dass der von den Franzosen auf 2000 Mann geschätzte laotische Widerstand sich in einer einzelnen Zone umgruppierte. Mendès-France wollte dem nicht stattgeben ${ }^{123}$, aber Eden schlug vor, eine einzelne Umgruppierungszone im Süden einzurichten. Dieser Vorschlag, auf den Zhou bewusst nicht einging, wurde schließlich angenommen. ${ }^{124}$ Als Ausgleich verlangte Zhou Sicherheitsgarantien für den Schut\% der Mitglieder der laotischen kommunistischen Widerstandsorganisation bei ihrer Rückkehr in ein normales Leben sowie die Möglichkeit für sie, sich in die Strukturen ihres Staates wieder eingliedern zu können. Mendès-France erklärte sich zwar bereit, die französischen Truppen auf 3000 Mann zu reduzieren, weigerte sich aber, einen Termin für die Evakuierung der Truppen aus Laos festzusetzen. Zum ersten Mal erklärte Zhou Enlai, dass er den Verbleib der französischen Truppen in Laos wünsche, damit sie den Laoten helfen, eine Nationalarmee aufzubauen. ${ }^{125}$ Am nächsten Tag, dem 20. Juli, setzte Zhou seine ganze Überzeugungskraft ein, um die Kambodschaner dazu zu überreden, ihre engen Beziehungen zu Frankreich zu wahren. Letztere weigerten sich im Gegensatz zu den Laoten, irgendeine Form von Abhängigkeit gegenüber ihrer ehemaligen Kolonialmacht aufrechtzuerhalten und wollten die Errichtung amerikanischer Basen in ihrem Lande. Zhou Enlai nahm nun einen diametral entgegengesetzten Standpunkt zu dem ein, was er während der ersten Verhandlungsphase ununterbrochen deklariert hatte, als er mit einer Vehemenz ohnegleichen „die französischen Kolonialherren für ihren Versuch anprangerte, die indochinesischen Völker in den Zustand des Sklaventums zurückversetzen zu wollen“. ${ }^{26}$

Am 20. Juli trafen Pham Van Dong und Mendès-France zweimal hintereinander zusammen. Mendès-France willigte ein, die Demarkationslinie entlang dem 17. Breitengrad festzulegen. Zuerst erfolgte seitens Pham Van Dongs keinerlei Reaktion. ${ }^{127}$ Schließlich akzeptierte er widerwillig: Der Übergang vom 16. zum 17. Breitengrad verpflichtete ihn, auf die Städte Danang und Hue, die kaiserliche Hauptstadt, sowie auf die Straße N. 9 zu verzichten, welche die Küstenstadt Quang Tri und die in Laos gelegene Stadt Savannakhet verband. Am 21.Juli gegen zwei Uhr morgens - „nach harten Verhandlungen und einigen überraschenden Kompromissvorschlägen, welche in letzter Minute von Molotov gemacht

121 AMAEC: Minutes, Conversation between Zhang Wentian and Harold Caccia, Second meeting of 19 July; Joyaux, La Chine et le règlement, S. 284

${ }_{122}$ Lưu Văn Lợi/Nguyễn Hồng Thạch, Pháp tái chiềm Đông Dương, S. 247.

12.3 AMAEC: Minutes, Conversation between Zhou Enlai, Pierre Mendès-France and Eden, 19 July 1954.

${ }^{124}$ AMAEC: Telegram, Zhou Enlai to Mao Zedong and others, regarding Zhou's meeting with MendèsFrance and Eden, and discussions outside the conference, 20 July 1954.

${ }^{125}$ AMAEC: Minutes, Conversation between Zhou Enlai, Pierre Mendès-France and Eden, 19 July 1954.

${ }^{126}$ ADF, s. Cabinet du Ministre, ss. Georges Bidault (1953-1954), d. 28, S. 231-250, hier S. 239: Compte rendu sténographique provisoire, daté du 14 mai 1954 , de la $3^{\text {imc }}$ séance plénière tenue au Palais des Nations à Genève le 12 mai 1954.

127 AMAEC: Telegram, Zhou Enlai to Mao Zedong and others, regarding Zhou's meeting with MendèsFrance and Eden, and discussions outside the conference, 20 July 1954, 12.00 pm. 
wurden“, waren Eden zufolge die letzten Streitthemen aus dem Weg geräumt. Molotov hatte akzeptiert, dass Kambodscha ein anderes Land - das heißt die Vereinigten Staaten um Hilfe bitten könne, sollte das Land von außen bedroht werden. Unter dem Druck von Mendès-France konzedierte Molotov Laos das gleiche Recht. ${ }^{128}$ Man kann sich fragen, was Molotov mit diesen beiden Konzessionen bezweckte, die dem diametral entgegengesetzt waren, wofür Zhou Enlai in den letzten Tagen mit solcher Beharrlichkeit eingetreten war: Wollte er einfach nur, dass die Verhandlung um jeden Preis zu einem Ergebnis führte und setzte deshalb sein ganzes Vertrauen in Übereinkünfte, bei denen die Gefahr bestand, dass sie von den Kambodschanern nicht unterzeichnet würden, oder suchte er bereits nach Wegen, wie er der aufkeimenden Macht Chinas Schranken setzen könnte?

\section{Fazit: Die Verratsanschuldigungen im Rückblick}

Am 22. Juli hielt Ho Chi Minh eine Ansprache, in der er der sowjetischen und der chinesischen Delegation herzlich dankte. ${ }^{129}$ Fernab von dieser idyllischen Vorstellung haben seitdem viele vietnamesische Historiker die Rolle Chinas - und jene der Sowjetunion - bei den Verhandlungen über das Genfer Abkommen kritisiert. Diese Kritik, die im Februar 1979 nach dem Einmarsch Chinas in die gebirgige Grenzregion Nordvietnams besonders virulent wurde, hält bis heute an, wenn auch in einer eher verschleierten Form. Die vietnamesische Historiographie wirft der chinesischen Delegation im Einzelnen vor, „die Tatsache ausgenützt zu haben, dass Frankreich sich weigerte, aus einer schwachen Verhandlungsstellung mit ihm [dem Viet Minh] Gespräche zu führen, um sich zu erlauben, mit Frankreich in direkte Besprechungen einzutreten und ein Abkommen über fundamentale Punkte mit ihm zu schließen“, ferner „bereits im Mai 1954 den 16. Breitengrad als Demarkationslinie vorgeschlagen und den Viet Minh dazu genötigt zu haben, dass die DRV auf die Stadt Hanoi, den Hafen von Hai Phong und auf die Straße, die diese beiden Städte verbindet, verzichtete“, und schließlich „mit der Ausweitung des Krieges unter der Rute der Amerikaner" gedroht zu haben, um auf Vietnam Druck auszuüben, insbesondere nach dem 10. Juli 1954“. ${ }^{130}$ Laut William Duiker gingen andere Historiker sogar so weit, China zu beschuldigen, dass es seine Einflusssphäre auf beide Länder auszudehnen versucht habe, indem es einerseits die Teilung Vietnams und andererseits den Abzug der Viet-Minh-Verbände aus Laos und Kambodscha billigte. ${ }^{131}$ Die Sachverhalte, die ich anhand französischer, chinesischer und russischer Archivquellen rekonstruiert habe, soweit ich diese konsultieren konnte, zeigen, dass die vorgebrachten Beschwerden nicht ungerechtfertigt sind, allerdings nur eine partielle Sicht der Ereignisse darstellen. Molotov und Zhou Enlai vereinten all ihre Bemühungen, um ein Friedensabkommen über Indochina zu erzielen: die Chinesen taten es aus Furcht vor einer Eskalation des Krieges und einem offenen Konflikt mit den USA, der in einen Atomkrieg hätte münden können; die Sowjets, weil sie Frankreich von der Last des indonesischen Krieges befreien und die Aufschiebung des Projekts des europäischen Verteidigungsvertrags erreichen wollten.

128 Gaiduk, Confronting Vietnam, S. 49.

129 Hồ Chí Minh, Tuyền tập, Hanoi, 3 Bde., Bd. 2 (1945-1954), S. 760-763.

130 Sự thật về quan hệ Việt Nam - Trung Quốc, S. 32.

131 Duiker, US Containment Policy, S. 182. 
Die noch am Tag der Unterzeichnung der Verträge in die Wege geleiteten Besprechungen Molotovs mit Mendès-France zeigen, dass die deutsche Frage in den Köpfen der sowjetischen Führung weiterhin das beherrschende Thema war. ${ }^{132}$ Mit Genugtuung registrierten die Sowjets, dass das EVG-Projekt im August 1954 von der Nationalversammlung abgelehnt wurde und leblos blieb. Vor diesem Hintergrund belegen die französischen Quellen auch, dass es während der Konferenz zwischen Molotov und Mendès-France zu keinen Geheimabsprachen gekommen ist: Molotov hatte seit dem 10. Juli Mendès-France vorgeschlagen, sich mit ihm über bestimmte Europa betreffende Fragen auszutauschen, was Letzterer ablehnte. Alles in allem änderten die Nachfolger Stalins nichts an den Prioritäten der sowjetischen Außenpolitik. Die von den Kommunisten geführten Dekolonisierungskriege blieben, ohne vernachlässigt zu werden, den europäischen Fragen untergeordnet. Die sowjetische Führung verfolgte diesen politischen Kurs zumindest bis in die 1960er Jahre weiter, als die Dritte Welt ein Feld der Rivalität mit China wurde.

Über die von den vietnamesischen Historikern gegen China gerichteten Verratsanschuldigungen lässt sich ebenfalls streiten. Es wurde gezeigt, dass Zhou Enlai, mit seinen Bemühungen, die Errichtung von amerikanischen Militärbasen in Laos und Kambodscha zu verhindern, dem Verbleib dieser beiden Länder im französischen Orbit immer offener Vorschub leistete. Während der Geheimverhandlungen, die er mit seinen französischen und britischen Amtskollegen führte, legte er einen Pragmatismus an den Tag, der in diametralem Gegensatz zu der ideologischen Rigidität stand, die er in den Plenarsitzungen zur Schau stellte. Eine Reihe von Indizien spricht im Übrigen dafür, dass er während der Genfer Konferenz starken Druck auf die Viet-Minh-Delegation übte. Auf der anderen Seite zeigen die Archive auch, dass die Chinesen nicht anstrebten, das Feld der Verhandlungen über das indochinesische Problem hinaus zu erweitern, noch irgendwelche Gegenleistungen für die Milderung ihrer Position zu erhalten. Dem indischen Botschafter Krishna Menon zufolge war Zhou Enlai, den er Ende Juni getroffen hatte, „bei weitem mehr vom Koreaproblem beherrscht als von allen anderen“ und „alle Indochina betreffende Lösungsvorschläge wurden von ihm danach beurteilt, wie sie als Präzedenzfall für die Koreafrage angewendet werden konnten “. ${ }^{133}$ Des Weiteren weiß man wegen der Unzugänglichkeit der vietnamesischen Archive weder über die exakte Position Bescheid, die der Viet Minh zu Beginn der Konferenz einnahm, noch wie sich diese Position im Weiteren entwickelt hat; die von den militärischen Viet-Minh-Delegierten abgegebenen Erklärungen weisen auf eine gewisse Bereitschaft hin, den Kampf fortzusetzen, sind aber nicht beweiskräftig.

Trotz des Scheiterns der Koreaverhandlungen steht die Genfer Indochinakonferenz für einen Sieg der sowjetischen Diplomatie. Die Konferenz, die auf das Drängen der Sowjets einberufen wurde, erlaubte es Moskau, seine Doktrin der „Friedlichen Koexistenz“ zu fördern und gleichzeitig die internationalen Spannungen zu reduzieren. Moskau hatte die Initiative; Washington war in der Defensive. In dem Propagandakrieg, den sich die beiden Großmächte lieferten, verstärkte die Sowjetunion das Bild von sich, das sie in der Weltöffentlichkeit durchsetzen wollte: das der Verkörperung des „Friedenslagers“. Schließlich war es ihr gelungen, das Auftreten Chinas auf der internationalen Bühne durchzusetzen.

\footnotetext{
132 ADF, s. Asie-Océanie (1945-1955), ss. Dossiers Généraux, d. 232, S. 237-243: Entretien de M. Mendès-France avec M. Molotov après le dîner du 10 juillet, fait à Genève le 12 juillet 1954 .

133 ADF, s. Cabinet du Ministre, ss. P. Mendès-France, d. 2, S. 16f.: Télégramme adressé de New York et

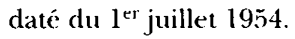


Entgegen den Vermutungen, die von manchen Historikern und zeitgenössischen politischen Akteuren angestellt wurden, standen die Beziehungen zwischen der Sowjetunion und China vor und nach der Konferenz unter dem Zeichen des Vertrauens und der Zusammenarbeit und nicht des Misstrauens und der Konkurrenz. Dennoch sollte man weder den Erfolg noch die Tragweite des Genfer Abkommens überschätzen. Die Lage zwischen China und den Vereinigten Staaten von Amerika blieb angespannt. Am 21.Juli verweigerte der stellvertretende Außenminister der USA - ebenso wie der Repräsentant Süd-Vietnams die Unterzeichnung des Abkommens, was ihn jedoch nicht daran hinderte, zu proklamieren, dass die Vereinigten Staaten jeglichen Vorstoß gegen das Abkommen als eine Bedrohung des Friedens und der internationalen Sicherheit betrachten würden. Die Amerikaner ergriffen auf der Stelle mehrere Maßnahmen zur Eindämmung der Expansion des Kommunismus in Südostasien: Sie setzten den proamerikanischen und antifranzösischen Nationalisten Ngo Dihn Diem an die Spitze der Regierung in Saigon und gründeten im September 1954 die SEATO, deren Schutzbereich - ungeachtet der im Genfer Abkommen vorgesehenen Neutralität - Laos, Kambodscha und Südvietnam einschloss. ${ }^{134}$ Ihrerseits traf die Volksrepublik China ab Ende Juli 1954 Vorkehrungen, die auf ihre Wiederbewaffnung und den Einmarsch in Taiwan abzielten. ${ }^{135}$ In den folgenden Jahren wurden mehrere entscheidende Punkte des Genfer Abkommens nicht eingehalten. 1956 weigerte sich die südvietnamesische Regierung, die Wahlen zu organisieren, welche der Wiedervereinigung des Landes vorangehen sollten. Im Januar 1959 beschloss die Partei der vietnamesischen Werktätigen insgeheim, den Vietnamkrieg wieder in Gang zu bringen. Im Monat darauf taten die Vereinigten Staaten das, was die Chinesen auf der Genfer Indochinakonferenz mit aller Kraft zu vermeiden versucht hatten: Sie errichteten in Laos einen Militärstützpunkt. Auch wenn sie keine Garantie für einen dauerhaften Frieden waren, rückten die Genfer Indochinaverträge das sowohl von Churchill und Eden als auch von Malenkov - der seinen Posten zugunsten Chruščevs verlor - beschworene Risiko eines dritten Weltkrieges in weitere Ferne. Da die Vereinigten Staaten im Oktober 1953 die Doktrin der massiven Vergeltung angenommen hatten, wäre dieser Konflikt sicherlich mit dem Einsatz von Nuklearwaffen verbunden gewesen. In dieser Hinsicht ist es denkbar, dass die gemeinsamen Bemühungen von Eden, Zhou Enlai, Molotov und Mendès-France, gleichgültig aus welcher Motivation heraus sie gehandelt haben mögen, nicht nur die - wenn auch vorübergehende - Wiederherstellung des Friedens in Indochina möglich gemacht haben. Sie waren vielleicht auch für die Welt ein Segen.

Aus dem Französischen übertragen von Marie-Thérèse Delteil

134 Yang Kuisong, Changes in Mao Zedong's Attitude toward the Indochina War, S. 12.

135 AMAEC: Telegram, CCP Central Committee to Zhou Enlai, concerning policies and measures in the struggle against the United States and (Chiang Kai-shek) after the Geneva Conference, 27 July 1954. 